\title{
Interval Logics with Applications to Study of Tense and Aspect in English
}

\author{
By \\ Hirokazu NishimuRA*
}

\section{Table of Contents}

\begin{tabular}{|c|c|}
\hline$\$ 1.1:$ & Introduction .................. \\
\hline$\S 1.2:$ & Temporal Dualism ......... \\
\hline Chapter 2: & The Propositional Logic I \\
\hline$\S 2.1$ : & Formal Language LI ...... \\
\hline$\S 2.2:$ & Semantics for I $\ldots \ldots \ldots$ \\
\hline$\S 2.3:$ & DI-sequence \\
\hline$\S 2.4:$ & Formal System I \\
\hline$\S 2.5:$ & 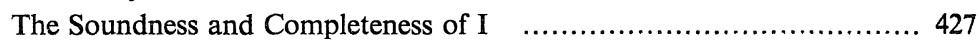 \\
\hline Chapter 3: & The Propositional Logic IM ..................... \\
\hline §3.1: & Formal Language LIM ........................... \\
\hline$\S 3.2:$ & Semantics for IM \\
\hline \$3.3: & DMI-sequence and DIM-sequence \\
\hline$\S 3.4:$ & Formal System IM ........................... \\
\hline$\S 3.5:$ & The Soundness and Completeness of IM \\
\hline Chapter 4 & 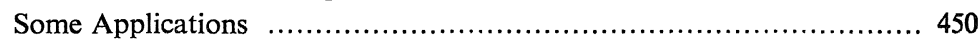 \\
\hline$\S 4.1$ & Some Interesting Tense Operators Definable within IM \\
\hline$\S 4.2:$ & 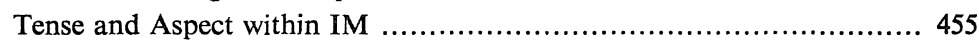 \\
\hline
\end{tabular}

\section{Chapter 1}

\section{Philosophical Motivation}

\section{§1.1. Introduction}

A common feature of traditional tense logics is that the truth-evaluation of a sentence is made at a moment of time or at best at a sequence of moments of time. The need of interval logics (i.e., tense logics which deal with sentences being true or false at intervals of time) has been stressed not only by so-called

Communicated by S. Takasu, July 10, 1978.

* Research Institute for Mathematical Sciences, Kyoto University. 
tense logicians but also by many researchers of linguistics, artificial intelligence, etc. either implicitly or explicitly. See, e.g., Bennett and Partee (1972, 1978), Bruce (1972), Dowty (1977), Gabbay (1976, p. 175), etc. Although many authors have discussed how to analyze several sample sentences since 1972 , there is no formal system of interval logic with an exact syntax and semantics comparable with traditional tense logics. For example, Bennett and Partee (1978, pp. 5354) have written as follows:

"One prominent inadequacy of the paper is that it does not provide a compositional syntax and semantics of the constructions treated... The truth conditions we gave for sample sentences of various types, even if correct, reflect the joint contribution of a host of such factors; separating out the individual contributions of the parts and how they fit together is a much harder task which remains to be done."

Bruce has stated as follows (1972, p. 24):

"Prior's tense logics are developed in terms of operators corresponding to "before" and "after". What tense logics result from operators corresponding to other time-segment relations?"

The main purpose of this paper is to answer these urgent requirements, i.e., we present some interval logics with exact syntax and semantics and establish their semantical completeness in this paper.

Our plan of the rest of this paper goes as follows. In the next section we present a minimal explanation on our own fundamental philosophical position, say, temporal dualism. The main interval logic which we advocate in this paper is IM, which will be discussed in Chapter 3. The interval logic I, which will be discussed in Chapter 2, is of interest, partly because it stands in the opposite extreme to traditional tense logics, and mainly because the formal discussion of I is to a considerable extent in parallelism to that of IM. In Chapter 4 we will show that many tense operators discussed in the literature are definable within our formal system IM. In this section we will give a sketch of how to deal with tenses in English in the formalism of IM.

\section{§1.2. Temporal Dualism}

The main purpose of this section is to give a brief explanation on our philosophical or linguistical position, called "temporal dualism", upon which our formal study of interval logics is to be based. Roughly speaking, temporal dualism means the idea that all sentences are classified into two classes, say those 
being true or false only at moments of time on the one hand, and those being true or false only at intervals of time on the other hand. A sentence of the former class is called an M-sentence, while a sentence of the latter class is called an I-sentence. Propositional variables should range only over sentences of the simple present tense, which we call atomic sentences, because sentences of other tenses should be constructed from such atomic sentences with the aid of relevant tense operators. An atomic sentence which is an M-sentence is called an s-sentence (" $\mathrm{s}$ " for "state (of affairs)"), because most of such sentences are the propositional counterparts of the notion of a state (of affairs) such as seen in von Wright (1963, chap. 2). Some examples of s-sentences are:

(1) Tom is in New York.

(2) Mary loves Tom.

(3) The window is open.

Practically we can identify s-sentences with sentences of the simple present tense which can not take the present progressive tense.

An atomic sentence which is an I-sentence is called a p-sentence (" $p$ " for "process"), because most of such sentences are the propositional counterparts of the notion of a process such as seen in Rescher and Urquhart (1971, chap. 14). Some examples of $\mathrm{p}$-sentences are:

(4) Susan walks in the park.

(5) Tom builds a house.

(6) It rains.

Practically we can identify p-sentences with sentences of the simple present tense which can take the present progressive tense. It is very interesting to notice that we usually do not utter such p-sentences, at least, in the reportive sense, as Bennett and Partee (1972) has stressed. By way of example, we usually say "it is raining now" rather than "it rains now". The present progressive tense operator, which we denote by ING, can apply exclusively to I-sentences and then yield $\mathrm{M}$-sentences. The sentences that we can utter in the reportive sense are only M-sentences.

Practically we can distinguish between M-sentences and I-sentences by considering whether the sentence at issue can take the present progressive tense. That is, if it can take the present progressive tense, then it must be an I-sentence. If it can not take the present progressive tense, then it must be an M-sentence. 
Therefore, as Bennett and Partee (1972) has suggested, all sentences of other tenses than the simple present tense that we can utter in the reportive tense are M-sentences. For example, the following sentences are M-sentences.

(7) Tom has built a house.

(8) Tom is building a house.

(9) Tom will build a house.

(10) Tom has been building a house.

(11) Tom will have built a house.

\section{Chapter 2}

\section{The Propositional Logic I}

\section{§ 2.1. Formal Language LI}

Definition 2.1.1. Our formal language LI consists of the following symbols:

(a) a countable set $\bar{P}$ of (propositional) variables: $\bar{p}, \bar{q}, \bar{p}^{\prime}, \ldots$;

(b) classical connectives: ᄀ, $\rightarrow$;

(c) tense operators: $I(n, x, y)$, where $n, x$ and $y$ range over all natural numbers such that $1 \leq x \leq y \leq n$;

(d) parentheses: $($,$) .$

Definition 2.1.2. The notion of a $w f f$ is defined by induction as follows:

(a) Any variable $\bar{p}$ is a wff.

(b) If $\bar{A}$ and $\bar{B}$ are wffs, so too are $(\neg \bar{A})$ and $(\bar{A} \rightarrow \bar{B})$.

(c) If for any $1 \leq i<j \leq n, \bar{A}_{i j}$ is a wff, so too is $I(n, x, y)\left(\bar{A}_{12}, \ldots, \bar{A}_{(n-1) n}\right)$, where $\bar{A}_{i j}$ 's are arranged in lexicographical order with respect to $(i, j)$.

In the rest of this paper we use parentheses in a very loose manner so as to make wffs easier to read. We should notice that $I(n, x, y)$ is a $\frac{n(n-1)}{2}$-ary tense operator. For the sake of notational simplicity, we will often write $I(n, x, y)\left(\left\{\bar{A}_{i j}\right\}_{1 \leq i<j \leq n}\right)$ or $I(n, x, y)\left(\left\{\bar{A}_{i j}\right\}\right)$ rather than $I(n, x, y)\left(\bar{A}_{12}, \ldots\right.$, $\left.\bar{A}_{(n-1) n}\right)$. We will make use of $I e(n, x, y)\left(\left\{\bar{A}_{i j}\right\}\right)$ as an abbreviation of $\neg I(n, x$, y) $\left(\left\{\neg A_{i j}\right\}\right)$, just as GA is an abbreviation of $\neg F \neg A$ in traditional tense logic. Symbols for conjunction $(\wedge)$, disjunction $(\vee)$, and material equivalence $(\leftrightarrow)$ are defined as usual. $\bar{T}$ and $\bar{F}$ are defined as abbreviations of $(\bar{p} \rightarrow \bar{p})$ and 
$\neg(\bar{p} \rightarrow \bar{p})$ for some variable $\bar{p}$ respectively. We will make use of ordinal notations in symbolic logic like $\wedge_{i=1}^{n} \bar{A}_{i}$ for $\left(\bar{A}_{1} \wedge\left(\bar{A}_{2} \wedge \cdots \wedge\left(\bar{A}_{(n-1)} \wedge \bar{A}_{n}\right) \cdots\right)\right)$ without any comment. We write $\bar{p}$ and $\bar{A}$ rather than $p$ and $A$ so as to stress that $\bar{p}$ and $\bar{A}$ represent propositions being truth-evaluated at intervals.

\section{§2.2. Semantics for $\mathbb{I}$}

Definition 2.2.1. A TI-structure is a triple $(S,<, D)$, where

(a) $S$ is a set (called the "time").

(b) $<$ is a strict linear order in $S$ (the earlier-later relation). That is, < is an asymmetric, transitive and connected binary relation having $S$ as its underlying set.

(c) $D$ is a function from $\bar{P} \times I(S,<)$ to $\{0,1\}$, where 1 stands for truth, 0 for falsity, and $I(S,<)=\{(s, t) \mid s<t$ and $s, t \in S\}$. That is, $D$ assigns a truth-value to each variable at each interval. We will often write $D(\bar{p}, s, t)$ rather than $D(\bar{p},(s, t))$.

Definition 2.2.2. Given a TI-structure $(S,<, D)$, the truth-value of a wff $\bar{A}$ at an interval $(s, t)$, notation: $\|\bar{A}\|_{(s, t)}$, is defined by induction as follows:

(a) $\|\bar{p}\|_{(s, t)}=D(\bar{p}, s, t)$, where $\bar{p}$ is a variable.

(b) $\|\neg \bar{A}\|_{(s, t)}=1$ iff $\|\bar{A}\|_{(s, t)}=0$.

(c) $\|\bar{A} \rightarrow \bar{B}\|_{(s, t)}=1$ iff $\|\bar{A}\|_{(s, t)}=0$ or $\|\bar{B}\|_{(s, t)}=1$.

(d) $\left\|I(n, x, y)\left(\left\{\bar{A}_{i j}\right\}\right)\right\|_{(s, t)}=1$ iff for some $s_{i}$ 's $(1 \leq i \leq n)$ such that $s_{1}<\cdots<$ $s_{n}, s_{x}=s$ and $s_{y}=t,\left\|\bar{A}_{i j}\right\|_{\left(s_{i}, s_{j}\right)}=1$ for any $(i, j)$.

With this definition, it is easy to see that:

(e) $\left\|\operatorname{Ie}(n, x, y)\left(\left\{\bar{A}_{i j}\right\}\right)\right\|_{(s, t)}=1$ iff for any $s_{i}$ 's $(1 \leq i \leq n)$ such that $s_{1}<\cdots<$ $s_{n}, s_{x}=s$ and $s_{y}=t,\left\|\bar{A}_{i j}\right\|_{\left(s_{i}, s_{j}\right)}=1$ for some $(i, j)$.

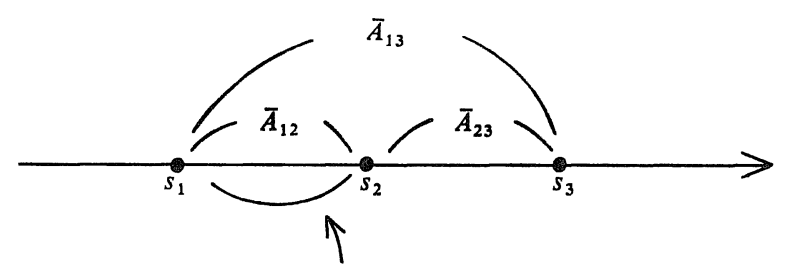

the distinguished reference interval

Figure 1. 


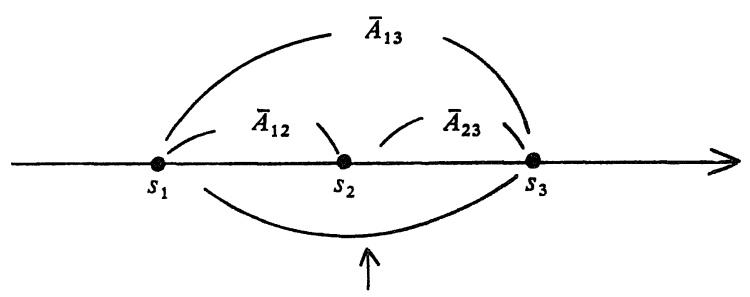

the distinguished reference interval

Figure 2.

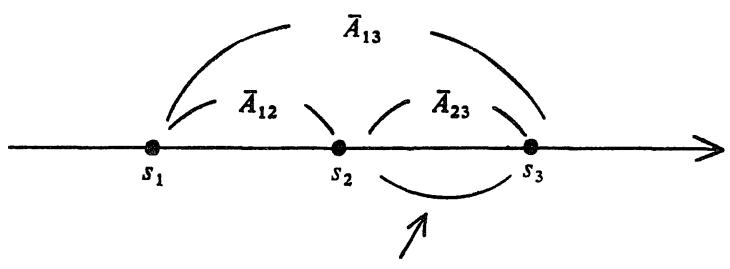

the distinguished reference interval

Figure 3.

By way of example, the situations depicted in Figures 1-3 are represented as follows:

(1) $I(3,1,2)\left(\bar{A}_{12}, \bar{A}_{13}, \bar{A}_{23}\right)$.

(2) $I(3,1,3)\left(\bar{A}_{12}, \bar{A}_{13}, \bar{A}_{23}\right)$.

(3) $I(3,2,3)\left(\bar{A}_{12}, \bar{A}_{13}, \bar{A}_{23}\right)$.

Definition 2.2.3. A TI-model is a 4-tuple $(S,<, D,(\bar{s}, \bar{t}))$, where

(a) $(S,<, D)$ is a TI-structure.

(b) $(\bar{s}, \bar{t}) \in I(S,<)$ (the distinguished reference interval).

A theory is a set of wffs.

\section{Definition 2.2.4.}

(1) A TI-model $\mu=(S,<, D,(\bar{s}, \bar{t}))$ is said to realize a wff $\bar{A}$, notation: $\mu \models \bar{A}$, if $\|\bar{A}\|_{(\bar{s}, \bar{t})}=1$.

(2) A TI-model $\mu=(S,<, D,(\bar{s}, \bar{t}))$ is said to realize a theory $\bar{\Sigma}$, notation: $\mu \models \bar{\Sigma}$, if $\|\bar{A}\|_{(\bar{s}, \bar{t})}=1$ for any $\bar{A} \in \bar{\Sigma}$.

(3) A wff $\bar{A}$ is said to be realizable if for some TI-model $\mu, \mu \vDash \bar{A}$.

(4) A theory $\bar{\Sigma}$ is said to be realizable if for some TI-model $\mu, \mu \models \bar{\Sigma}$. 
(5) A wff $\bar{A}$ is said to be valid, notation: $\vDash \bar{A}$, if $\mu \vDash \bar{A}$ for any TI-model $\mu$.

The rest of this section is devoted to some examples of valid wffs.

Example 2.2.5. The following wffs are valid.

(1) $I(4,2,3)\left(\bar{A}_{12}, \bar{A}_{13}, \bar{A}_{14}, \bar{A}_{23}, \bar{A}_{24}, \bar{A}_{34}\right) \rightarrow I(3,1,2)\left(\bar{A}_{23}, \bar{A}_{24}, \bar{A}_{34}\right)$.

(2) $I(4,1,3)\left(\bar{A}_{12}, \bar{A}_{13}, \bar{A}_{14}, \bar{A}_{23}, \bar{A}_{24}, \bar{A}_{34}\right) \rightarrow I(3,1,2)\left(\bar{A}_{13}, \bar{A}_{14}, \bar{A}_{34}\right)$.

Example 2.2.6. The following wff is valid.

$$
I(3,1,2)\left(\bar{A}_{12}, \bar{A}_{13}, \bar{A}_{23}\right) \rightarrow \bar{C}_{1} \vee \bar{C}_{2} \vee \bar{C}_{3},
$$

where

(a) $\bar{A}_{12}=I(3,1,2)\left(\bar{A}_{12}^{\prime}, \bar{A}_{13}^{\prime}, \bar{A}_{23}^{\prime}\right)$.

(b) $\bar{C}_{1}=I(4,1,2)\left(\bar{A}_{12} \wedge \bar{A}_{12}^{\prime}, \bar{A}_{13}^{\prime}, \bar{A}_{13}, \bar{A}_{23}^{\prime}, \bar{A}_{23}, \bar{T}\right)$.

(c) $\bar{C}_{2}=I(3,1,2)\left(\bar{A}_{12} \wedge \bar{A}_{12}^{\prime}, \bar{A}_{13} \wedge \bar{A}_{13}^{\prime}, \bar{A}_{23} \wedge \bar{A}_{23}^{\prime}\right)$.

(d) $\bar{C}_{3}=I(4,1,2)\left(\bar{A}_{12} \wedge \bar{A}_{12}^{\prime}, \bar{A}_{13}, \bar{A}_{13}^{\prime}, \bar{A}_{23}, \bar{A}_{23}^{\prime}, \bar{T}\right)$.

Example 2.2.7. The following wff is valid.

$$
I(3,1,2)\left(\bar{A}_{12}, \bar{A}_{13}, \bar{A}_{23}\right) \rightarrow \bar{C}_{1} \vee \bar{C}_{2} \vee \bar{C}_{3},
$$

where

(a) $\bar{A}_{12}=\operatorname{Ie}(3,1,2)\left(\bar{A}_{12}^{\prime}, \bar{A}_{13}^{\prime}, \bar{A}_{23}^{\prime}\right)$.

(b) $\quad \bar{C}_{1}=I(3,1,2)\left(\bar{A}_{12} \wedge \bar{A}_{12}^{\prime}, \bar{A}_{13}, \bar{A}_{23}\right)$.

(c) $\bar{C}_{2}=I(3,1,2)\left(\bar{A}_{12}, \bar{A}_{13} \wedge \bar{A}_{13}^{\prime}, \bar{A}_{23}\right)$.

(d) $\quad \bar{C}_{3}=I(3,1,2)\left(\bar{A}_{12}, \bar{A}_{13}, \bar{A}_{23} \wedge \bar{A}_{23}^{\prime}\right)$.

\section{§2.3. DI-Sequence}

This section is devoted to the definitions around the notion of a DI-sequence, which will be of importance to us.

\section{Definition 2.3.1.}

(1) By a DI-set we mean a triple $(S,<,(\bar{s}, \bar{t}))$, where

(a) $S$ is a set.

(b) $<$ is a strict linear order in $S$.

(c) $(\bar{s}, \bar{t})$ is an interval of $(S,<)$, i.e., $\bar{s}, \bar{t} \in S$ and $\bar{s}<\bar{t}$.

(2) A DI-set $\left(S^{\prime},<,(\bar{s}, \bar{t})\right)$ is called a DI-subset of a DI-set $(S,<,(\bar{s}, \bar{t}))$ if $\left(S^{\prime},<\right)$ is a restriction of $(S,<)$ as an ordered set, i.e., $S^{\prime}$ is a subset of $S$ and $s^{\prime}<t^{\prime}$ in $S^{\prime}$ iff $s^{\prime}<t^{\prime}$ in $S$ for any $s^{\prime}, t^{\prime} \in S^{\prime}$.

(3) A DI-set $(S,<,(\bar{s}, \bar{t}))$ is called canonical if $S=[1, n]=\{m \mid m$ is a natural 
number such that $1 \leq m \leq n\}$ for some $n$ and $<$ is the usual ordering of natural numbers.

\section{Definition 2.3.2.}

(1) A DI-monomorphism from a DI-set $(S,<,(\bar{s}, \bar{t}))$ to a DI-set $\left(S^{\prime},<\right.$, $\left.\left(\bar{s}^{\prime}, \bar{t}^{\prime}\right)\right)$ is an order monomorphism $f$ from $(S,<)$ to $\left(S^{\prime},<\right)$ such that $f(\bar{s})=\bar{s}^{\prime}$ and $f(\bar{t})=\bar{t}^{\prime}$.

(2) A DI-isomorphism from a DI-set $(S,<,(\bar{s}, \bar{t}))$ to a DI-set $\left(S^{\prime},<,\left(\bar{s}^{\prime}, \bar{t}^{\prime}\right)\right)$ is a DI-monomorphism from $(S,<,(\bar{s}, \bar{t}))$ onto $\left(S^{\prime},<,\left(\bar{s}^{\prime}, \bar{t}^{\prime}\right)\right)$.

(3) Two DI-sets $(S,<,(\bar{s}, \bar{t}))$ and $\left(S^{\prime},<,\left(\bar{s}^{\prime}, \bar{t}^{\prime}\right)\right)$ are called isomorphic, notation: $(S,<,(\bar{s}, \bar{t})) \approx\left(S^{\prime},<,\left(\bar{s}^{\prime}, \bar{t}^{\prime}\right)\right)$, if there exists a DI-isomorphism from one to the other.

\section{Definition 2.3.3.}

(1) A DI-sequence $\alpha$ is a 4-tuple $(S,<,(\bar{s}, \bar{t}), \Omega)$, where

(a) $(S,<,(\bar{s}, \bar{t}))$ is a DI-set.

(b) $\Omega$ is a function from $I(S,<)$ to WFF, where $I(S,<)=\{(s, t) \mid s, t \in S$ and $s<t\}$ and WFF is the totality of wffs. That is, $\Omega$ assigns a wff to each interval. We will often write $\Omega(s, t)$ rather than $\Omega((s, t))$.

(2) A DI-sequence $\left(S^{\prime},<,(\bar{s}, \bar{t}), \Omega^{\prime}\right)$ is called a DI-subsequence of a DIsequence $(S,<,(\bar{s}, \bar{t}), \Omega)$ if $\left(S^{\prime},<,(\bar{s}, \bar{t})\right)$ is a DI-subset of $(S,<,(\bar{s}, \bar{t}))$ and $\Omega^{\prime}$ is the restriction of $\Omega$ to $I\left(S^{\prime},<\right)$.

(3) A DI-sequence $(S,<,(\bar{s}, \bar{t}), \Omega)$ is called canonical if $(S,<,(\bar{s}, \bar{t}))$ is canonical as a DI-set.

The notion of a DI-subsequence will be very useful to study such situations as that in Example 2.2.5 from a general viewpoint.

\section{Definition 2.3.4.}

(1) A DI-monomorphism from a DI-sequence $(S,<,(\bar{s}, \bar{t}), \Omega)$ to a DI-sequence $\left(S^{\prime},<,\left(\bar{s}^{\prime}, \bar{t}^{\prime}\right), \Omega^{\prime}\right)$ is a DI-monomorphism $f$ from $(S,<,(\bar{s}, \bar{t}))$ to $\left(S^{\prime},<\right.$, $\left.\left(\bar{s}^{\prime}, \bar{t}^{\prime}\right)\right)$ such that $\Omega=\Omega^{\prime} \circ f$, i.e., $\Omega(s, t)=\Omega^{\prime}(f(s), f(t))$ for any $(s, t) \in I(S,<)$.

(2) A DI-monomorphism $f$ is called a DI-isomorphism if $f$ is onto.

(3) Two DI-sequences $(S,<,(\bar{s}, \bar{t}), \Omega)$ and $\left(S^{\prime},<,\left(\bar{s}^{\prime}, \bar{t}^{\prime}\right), \Omega^{\prime}\right)$ are called isomorphic, notation: $(S,<,(\bar{s}, \bar{t}), \Omega) \approx\left(S^{\prime},<,\left(\bar{s}^{\prime}, \bar{t}^{\prime}\right), \Omega^{\prime}\right)$, if there exists a DI-isomorphism from one to the other.

Definition 2.3.5. Let $\alpha=(S,<,(\bar{s}, \bar{t}), \Omega)$ and $\alpha^{\prime}=\left(S^{\prime},<,\left(\bar{s}^{\prime}, \bar{t}^{\prime}\right), \Omega^{\prime}\right)$ be 
DI-sequences and $\left(s_{1}, t_{1}\right) \in I(S,<)$. Then a sum of $\alpha$ and $\alpha^{\prime}$ with respect to $\left(s_{1}, t_{1}\right)$ is a triple $\left(\alpha^{\prime \prime}, f, g\right)$ (or simply, $\left.\alpha^{\prime \prime}\right)$, where

(a) $\alpha^{\prime \prime}$ is a DI-sequence $\left(S^{\prime \prime},<,\left(\bar{s}^{\prime \prime}, \bar{t}^{\prime \prime}\right), \Omega^{\prime \prime}\right)$.

(b) $f$ is a DI-monomorphism from $(S,<,(\bar{s}, \bar{t}))$ to $\left(S^{\prime \prime},<,\left(\bar{s}^{\prime \prime}, \bar{t}^{\prime \prime}\right)\right)$.

(c) $g$ is an order monomorphism from $\left(S^{\prime},<\right)$ to $\left(S^{\prime \prime},<\right)$ such that $g\left(\bar{s}^{\prime}\right)$ $=f\left(s_{1}\right)$ and $g\left(\bar{t}^{\prime}\right)=f\left(t_{1}\right)$.

(d) $S^{\prime \prime}=f(S) \cup g\left(S^{\prime}\right)$.

(e) (i) in the case $\left(s^{\prime \prime}, t^{\prime \prime}\right) \in I\left(S^{\prime \prime},<\right)$ is such that $\left(s^{\prime \prime}, t^{\prime \prime}\right)=(f(s), f(t))$ $=\left(g\left(s^{\prime}\right), g\left(t^{\prime}\right)\right)$ for some $(s, t) \in I(S,<)$ and $\left(s^{\prime}, t^{\prime}\right) \in I\left(S^{\prime},<\right)$ : $\Omega^{\prime \prime}\left(s^{\prime \prime}, t^{\prime \prime}\right)=\Omega(s, t) \wedge \Omega^{\prime}\left(s^{\prime}, t^{\prime}\right)$, i.e., $\Omega^{\prime \prime}\left(s^{\prime \prime}, t^{\prime \prime}\right)$ is the conjunction of $\Omega(s, t)$ and $\Omega^{\prime}\left(s^{\prime}, t^{\prime}\right)$.

(ii) in the case $\left(s^{\prime \prime}, t^{\prime \prime}\right) \in I\left(S^{\prime \prime},<\right)$ is such that $\left(s^{\prime \prime}, t^{\prime \prime}\right)=(f(s), f(t))$ for some $(s, t) \in I(S,<)$ and $\left(s^{\prime \prime}, t^{\prime \prime}\right) \neq\left(g\left(s^{\prime}\right), g\left(t^{\prime}\right)\right)$ for any $\left(s^{\prime}, t^{\prime}\right)$ $\in I\left(S^{\prime},<\right): \Omega^{\prime \prime}\left(s^{\prime \prime}, t^{\prime \prime}\right)=\Omega(s, t)$.

(iii) in the case $\left(s^{\prime \prime}, t^{\prime \prime}\right) \in I\left(S^{\prime \prime},<\right)$ is such that $\left(s^{\prime \prime}, t^{\prime \prime}\right) \neq(f(s), f(t))$ for any $(s, t) \in I(S,<)$ and $\left(s^{\prime \prime}, t^{\prime \prime}\right)=\left(g\left(s^{\prime}\right), g\left(t^{\prime}\right)\right)$ for some $\left(s^{\prime}, t^{\prime}\right)$ $\in I\left(S^{\prime},<\right): \Omega^{\prime \prime}\left(s^{\prime \prime}, t^{\prime \prime}\right)=\Omega^{\prime}\left(s^{\prime}, t^{\prime}\right)$.

(iv) otherwise: $\Omega^{\prime \prime}\left(s^{\prime \prime}, t^{\prime \prime}\right)=\bar{T}$.

We denote by $\operatorname{CS}\left(\alpha, \alpha^{\prime}, s_{1}, t_{1}\right)$ the totality of sums of $\alpha$ and $\alpha^{\prime}$ with respect to $\left(s_{1}, t_{1}\right)$ that are canonical. It is important to notice that $\operatorname{CS}\left(\alpha, \alpha^{\prime}, s_{1}, t_{1}\right)$ is a finite set provided $\alpha$ and $\alpha^{\prime}$ are finite (i.e., the cardinalities of $S$ and $S^{\prime}$ are finite).

The notion of a sum will be very useful in dealing with such situations as that in Example 2.2.6 from a general point.

Definition 2.3.6. Let $\alpha=(S,<,(\bar{s}, \bar{t}), \Omega)$ and $\alpha^{\prime}=\left(S^{\prime},<,\left(\bar{s}^{\prime}, \bar{t}^{\prime}\right), \Omega^{\prime}\right)$ be DI-sequences and $f$ be an order monomorphism from $\left(S^{\prime},<\right)$ to $(S,<)$. Then the immersion of $\alpha^{\prime}$ into $\alpha$ at $\left(s_{1}^{\prime}, t_{1}^{\prime}\right) \in I\left(S^{\prime},<\right)$ with respect to $f$ is the DI-sequence $\alpha^{\prime \prime}=\left(S,<,(\bar{s}, \bar{t}), \Omega^{\prime \prime}\right)$, where $\Omega^{\prime \prime}\left(f\left(s_{1}^{\prime}\right), f\left(t_{1}^{\prime}\right)\right)=\Omega\left(f\left(s_{1}^{\prime}\right), f\left(t_{1}^{\prime}\right)\right) \wedge \Omega^{\prime}\left(s_{1}^{\prime}, t_{1}^{\prime}\right)$ while $\Omega^{\prime \prime}(s, t)=\Omega(s, t)$ for any other $(s, t) \in I(S,<)$. We denote by $\operatorname{IM}\left(\alpha, \alpha^{\prime}, f\right)$ the totality of immersions of $\alpha$ into $\alpha^{\prime}$ with respect to $f$.

The notion of an immersion will be useful in dealing with such situations as that in Example 2.2.7 from a general point.

\section{§2.4. Formal System I}

The main purpose of this section is to present the formal system I. To do 
it smoothly, we need some notational conventions.

Given a finite DI-sequence $\alpha=(S,<,(\bar{s}, \bar{t}), \Omega)$, we can easily find out the canonical DI-sequence $\alpha^{\prime}=\left([1, n],<,(1, r), \Omega^{\prime}\right)$ such that $\alpha^{\prime} \approx \alpha$. We will denote the wff $I(n, x, y)\left(\left\{\Omega^{\prime}(i, j)\right\}\right)$ by $I(n, x, y)(\alpha)$ or $I(,),(\alpha)$. That is,

$$
I(n, x, y)(\alpha)=I(,,)(\alpha)=I(n, x, y)\left(\left\{\Omega^{\prime}(i, j)\right\}\right) .
$$

$\operatorname{Ie}(n, x, y)(\alpha)$ or $\operatorname{Ie}(,),(\alpha)$ is defined similarly.

Definition 2.4.1. Our formal system I consists of the following (inference) rules and axioms (or exactly, axiom schemata):

Rules:

$$
\begin{array}{ll}
\text { (MP) } & \frac{\vdash \bar{A} \quad \vdash \bar{A} \rightarrow \bar{B}}{\vdash \bar{B}} \\
\text { (RID) } & \frac{\vdash \bar{A}_{i j} \leftrightarrow \bar{B}_{i j} \quad(1 \leq i<j \leq n)}{\vdash I(n, x, y)\left(\left\{\bar{A}_{i j}\right\}\right) \leftrightarrow I(n, x, y)\left(\left\{\bar{B}_{i j}\right\}\right)} \\
\text { (RIN) } & \frac{\vdash \bar{A}}{\vdash I e(n, x, y)\left(\left\{\bar{A}_{i j}\right\}\right)},
\end{array}
$$

where $\bar{A}_{i j}=\bar{A}$ for some $(i, j)$.

Axioms:

(A1) $\bar{A} \rightarrow(\bar{B} \rightarrow \bar{A})$

(A2) $(\bar{A} \rightarrow(\bar{B} \rightarrow \bar{C})) \rightarrow((\bar{A} \rightarrow \bar{B}) \rightarrow(\bar{A} \rightarrow \bar{C}))$

(A3) $(\neg \bar{B} \rightarrow \neg \bar{A}) \rightarrow(\bar{A} \rightarrow \bar{B})$

(I0) $I(2,1,2)(\bar{T})$

(I1) $I e(n, x, y)\left(\left\{\bar{A}_{i j}\right\}\right) \wedge I e(n, x, y)\left(\left\{\bar{A}_{i j}^{\prime}\right\}\right) \rightarrow \operatorname{Ie}(n, x, y)\left(\left\{\bar{A}_{i j}^{\prime \prime}\right\}\right)$, where for some $\left(i_{1}, j_{1}\right), \bar{A}_{i_{1} j_{1}}=\bar{A}_{i_{1} j_{1}}^{\prime} \rightarrow \bar{A}_{i_{1} j_{1}}^{\prime \prime}$, while for any other $(i, j), \bar{A}_{i j}=\bar{A}_{i j}^{\prime}=\bar{A}_{i j}^{\prime \prime}$.

(I2) $I(n, x, y)\left(\left\{\bar{A}_{i j}\right\}\right) \leftrightarrow B \wedge I(n, x, y)\left(\left\{\bar{A}_{i j}^{\prime}\right\}\right)$, where $A_{x y}=B \wedge A_{x y}^{\prime}$, while $A_{i j}=A_{i j}^{\prime}$ for any other $(i, j)$.

(I3) $I(,),(\alpha) \rightarrow I(,),\left(\alpha^{\prime}\right)$, where $\alpha^{\prime}$ is a DI-subsequence of $\alpha$.

(I4) $I(n, x, y)\left(\left\{\bar{A}_{i j}\right\}\right) \rightarrow \vee_{\beta \in C S\left(\alpha, \alpha^{\prime}, i_{1}, j_{1}\right)} I(,),(\beta)$, where
(a) for some $1 \leq i_{1}<j_{1} \leq n$, $\bar{A}_{i_{1} j_{1}}=I\left(n^{\prime}, x^{\prime}, y^{\prime}\right)\left(\left\{\bar{A}_{i j}^{\prime}\right\}\right)$.
(b) $\alpha=([1, n],<,(x, y), \Omega)$, where 


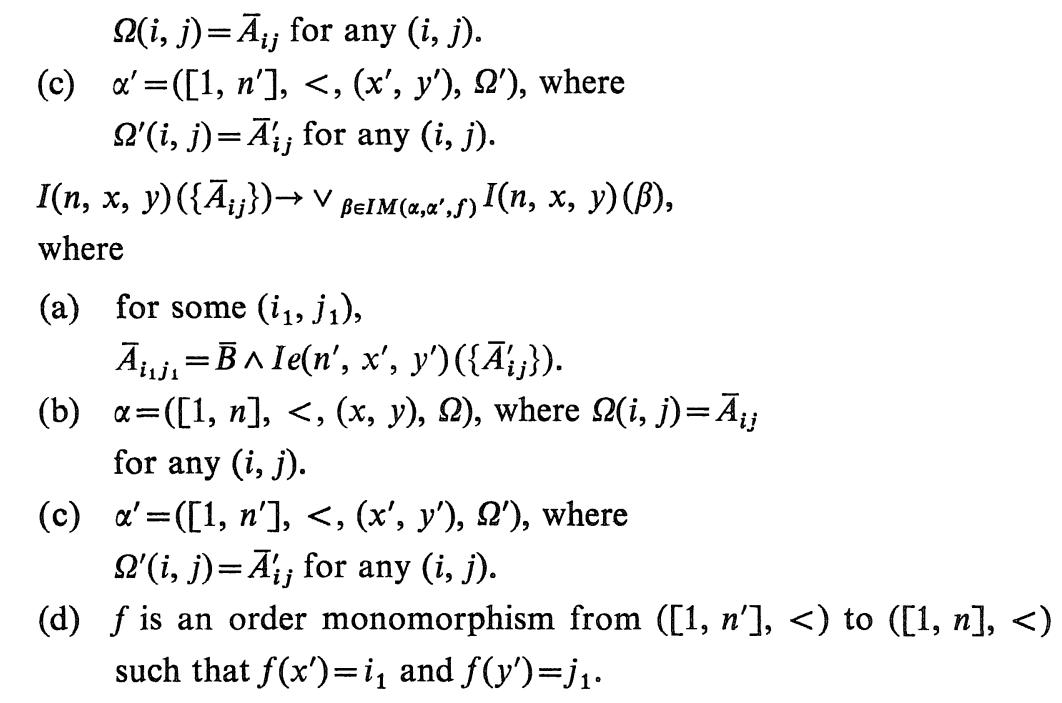

Some explanation on the above list of axioms and rules is in order. The axioms (A1)-(A3) and the rule (MP) constitute classical propositional calculus (hereafter called PC). The axioms (I0)-(I2) and the rules (RID) and (RIN) are concerned with each tense operator as such.

By the way, it is very interesting and instructive to notice that each tense operator $\operatorname{Ie}(n, x, y)$, if separated completely from other tense operators, is very similar to that of Gabbay's system $E^{[n]}$. As for $E^{[n]}$, see Gabby (1976, pp. 184-186). In this sense our formal system I may be regarded as an interconnected family of variants of $E^{[n]}$ 's. The axioms (I3)-(I5) deal with the relationship of tense operators. The axiom (I3) deals with such situations as that in Example 2.2.5 generally. The axioms (I4) and (I5) deal with so-called iterations of tense operators. The axiom (I4) deals with such situations as that in Example 2.2.6 generally. This axiom states what logical conclusion follows from wffs of the form $I(n, x, y)\left(\left\{\bar{A}_{i j}\right\}\right)$, an argument $\bar{A}_{i j}$ of which is again of this form. The axiom (I5) deals with such situations as that in Example 2.2.7 generally. That is, the axiom (I5) states what logical conclusion follows from wffs of the form $I(n, 1, r)\left(\left\{\bar{A}_{i j}\right\}\right)$, an argument $\bar{A}_{i, j}$ of which is in turn of the form $\operatorname{Ie}\left(n^{\prime}, 1^{\prime}, r^{\prime}\right)\left(\left\{\bar{A}_{i j}^{\prime}\right\}\right)$.

\section{\$2.5. The Soundness and Completeness of 1}

Theorem 2.5.1 (Soundness of I). For any wff $\bar{A}$, if $\vdash \bar{A}$, then $\vDash \bar{A}$.

Proof. By induction on the construction of the proof. I.e., it is sufficient 
to prove that all the axioms of I are valid and the rules of I preserve validity. Since it is almost straight, it is left to the reader as an exercise.

The rest of this section is devoted thoroughly to the proof of the following theorem.

Theorem 2.5.2 (Completeness of I). A theory $\bar{\Sigma}$ is consistent iff it is realizable.

Corollary 2.5.3. For any wff $\bar{A}, \vdash \bar{A}$ iff $\vDash \bar{A}$.

Here in order to establish the strong completeness of I, we will use a mixture of the tableaux method and the so-called classical Henkin method (say, Henkin (1949)). To do it smoothly, several definitions and lemmas are in order.

Now we define several notions around that of a TDI-sequence. Very roughly, a DI-sequence was a DI-set with an assignment of wffs to intervals, while a TDI-sequence is a DI-set with an assignment of theories (i.e., sets of wffs) to intervals.

\section{Definition 2.5.4.}

(1) A TDI-sequence $\lambda$ is a 4-tuple $(S,<,(\bar{s}, \bar{t}), \Psi)$, where

(a) $(S,<,(\bar{s}, \bar{t}))$ is a DI-set.

(b) $\Psi$ is a function from $I(S,<)$ to $2^{\mathrm{WFF}}$, where $2^{\mathrm{WFF}}$ is the power set of WFF. That is, $\Psi$ assigns a theory $\Psi(s, t)$ to each interval $(s, t)$.

(2) A TDI-sequence $\lambda^{\prime}=\left(S^{\prime},<,(\bar{s}, \bar{t}), \Psi^{\prime}\right)$ is called a TDI-subsequence of a TDI-sequence $\lambda=(S,<,(\bar{s}, \bar{t}), \Psi)$, notation: $\lambda^{\prime} \subseteq \lambda$, if $\left(S^{\prime},<,(\bar{s}, \bar{t})\right)$ is a DI-subset of $(S,<,(s, t))$ and $\Psi^{\prime}(s, t) \subseteq \Psi(s, t)$ for any $(s, t) \in I\left(S^{\prime},<\right)$, where $\subseteq$ is the set inclusion.

(3) A TDI-sequence $(S,<,(\bar{s}, \bar{t}), \Psi)$ is called finite if $S$ is a finite set and $\Psi(s, t)$ is also a finite set for any $(s, t) \in I(S,<)$.

(4) A TDI-sequence $(S,<,(\bar{s}, \bar{t}), \Psi)$ is called canonical if $(S,<,(\bar{s}, \bar{t}))$ is canonical as a DI-set.

Definition 2.5.5. Let $\lambda=(S,<,(\bar{s}, \bar{t}), \Psi)$ and $\lambda^{\prime}=\left(S^{\prime},<,\left(\bar{s}^{\prime}, \bar{t}^{\prime}\right), \Psi^{\prime}\right)$ be TDI-sequences and $\left(s_{1}, t_{1}\right) \in I(S,<)$. Then a sum of $\lambda$ and $\lambda^{\prime}$ with respect to $\left(s_{1}, t_{1}\right)$ is a triple $\left(\lambda^{\prime \prime}, f, g\right)$ (or simply, $\left.\lambda^{\prime \prime}\right)$, where

(a) $\lambda^{\prime \prime}$ is a TDI-sequence $\left(S^{\prime \prime},<,\left(\bar{s}^{\prime \prime}, \bar{t}^{\prime \prime}\right), \Psi^{\prime \prime}\right)$.

(b) $f$ is a DI-monomorphism from $(S,<,(\bar{s}, \bar{t}))$ to $\left(S^{\prime \prime},<,\left(\bar{s}^{\prime \prime}, \tilde{t}^{\prime \prime}\right)\right)$.

(c) $g$ is an order monomorphism from $\left(S^{\prime},<\right)$ to $\left(S^{\prime \prime},<\right)$ such that $g\left(\bar{s}^{\prime}\right)$ 
$=f\left(s_{1}\right)$ and $g\left(\tilde{t}^{\prime}\right)=f\left(t_{1}\right)$.

(d) $S^{\prime \prime}=f(S) \cup g\left(S^{\prime}\right)$.

(e) $\Psi^{\prime \prime}$ is defined as follows:

(i) In the case $\left(s^{\prime \prime}, t^{\prime \prime}\right) \in I\left(S^{\prime \prime},<\right)$ is such that $\left(s^{\prime \prime}, t^{\prime \prime}\right)=(f(s), f(t))$ $=\left(g\left(s^{\prime}\right), g\left(t^{\prime}\right)\right)$ for some $(s, t) \in I(S,<)$ and $\left(s^{\prime}, t^{\prime}\right) \in I\left(S^{\prime},<\right)$ : $\Psi^{\prime \prime}\left(s^{\prime \prime}, t^{\prime \prime}\right)=\Psi(s, t) \cup \Psi^{\prime}\left(s^{\prime}, t^{\prime}\right)$, i.e., $\Psi^{\prime \prime}\left(s^{\prime \prime}, t^{\prime \prime}\right)$ is the union of $\Psi(s, t)$ and $\Psi^{\prime}\left(s^{\prime}, t^{\prime}\right)$.

(ii) In the case $\left(s^{\prime \prime}, t^{\prime \prime}\right) \in I\left(S^{\prime \prime},<\right)$ is such that $\left(s^{\prime \prime}, t^{\prime \prime}\right)=(f(s), f(t))$ for some $(s, t) \in I(S,<)$ and $\left(s^{\prime \prime}, t^{\prime \prime}\right) \neq\left(g\left(s^{\prime}\right), g\left(t^{\prime}\right)\right)$ for any $\left(s^{\prime}, t^{\prime}\right)$ $\in I\left(S^{\prime},<\right): \Psi^{\prime \prime}\left(s^{\prime \prime}, t^{\prime \prime}\right)=\Psi(s, t)$.

(iii) In the case $\left(s^{\prime \prime}, t^{\prime \prime}\right) \in I\left(S^{\prime \prime},<\right)$ is such that $\left(s^{\prime \prime}, t^{\prime \prime}\right) \neq(f(s), f(t))$ for any $(s, t) \in I(S,<)$ and $\left(s^{\prime \prime}, t^{\prime \prime}\right)=\left(g\left(s^{\prime}\right), g\left(t^{\prime}\right)\right)$ for some $\left(s^{\prime}, t^{\prime}\right)$ $\in I\left(S^{\prime},<\right): \Psi^{\prime \prime}\left(s^{\prime \prime}, t^{\prime \prime}\right)=\Psi^{\prime}\left(s^{\prime}, t^{\prime}\right)$.

(iv) Otherwise: $\Psi^{\prime \prime}\left(s^{\prime \prime}, t^{\prime \prime}\right)=\emptyset$.

Definition 2.5.6. Suppose that:

(a) $\lambda=(S,<,(\bar{s}, \bar{t}), \Psi)$ is a TDI-sequence.

(b) $\alpha^{\prime}=\left(S^{\prime},<,\left(\bar{s}^{\prime}, \bar{t}^{\prime}\right), \Omega^{\prime}\right)$ is a DI-sequence.

(c) $f$ is an order monomorphism from $\left(s^{\prime},<\right)$ to $(S,<)$.

Then the immersion of $\alpha^{\prime}$ into $\lambda$ at $\left(s_{1}^{\prime}, t_{1}^{\prime}\right) \in I\left(S^{\prime},<\right)$ with respect to $f$ is the TDI-sequence $\lambda^{\prime \prime}=\left(S,<,(\bar{s}, \bar{t}), \Psi^{\prime \prime}\right)$, where $\Psi^{\prime \prime}\left(f\left(s_{1}^{\prime}\right), f\left(t_{1}^{\prime}\right)\right)=\Psi\left(f\left(s_{1}^{\prime}\right), f\left(t_{1}^{\prime}\right)\right) \cup$ $\left\{\Omega^{\prime}\left(s_{1}^{\prime}, t_{1}^{\prime}\right)\right\}$ while $\Psi^{\prime \prime}(s, t)=\Psi(s, t)$ for any other $(s, t) \in I(S,<)$.

Given a finite TDI-sequence $\lambda=(S,<,(\bar{s}, \bar{t}), \Psi)$, we can define its associated DI-sequence $\alpha=(S,<,(\bar{s}, \bar{t}), \Omega)$, where $\Omega(s, t)=\wedge_{\bar{A} \in \Psi(s, t)} \bar{A}$ for any $(s, t)$ $\in I(S,<)$. I.e., $\Omega(s, t)$ is the conjunction of all the wffs of $\Psi(s, t)$. Especially, when $\Psi(s, t)$ is empty, $\Psi(s, t)=\bar{T}$ by convention. We define the wff $I(n, x, y)(\lambda)$ $=I(,),(\lambda)$ as $I(,),(\alpha)$.

Lemma 2.5.7. Suppose that:

(a) $\bar{\Sigma}$ is a theory and $\bar{A} \in \bar{\Sigma}$.

(b) $\lambda=(S,<,(\bar{s}, \bar{t}), \Psi)$ is a finite TDI-sequence.

(c) $\bar{\Sigma} \cup\{I(,),(\lambda)\}$ is consistent.

We define the TDI-sequence $\lambda^{\prime}=\left(S,<,(\bar{s}, \bar{t}), \Psi^{\prime}\right)$, where $\Psi^{\prime}(s, t)=\{\bar{A}\} \cup \Psi(s, t)$ while $\Psi(s, t)=\Psi^{\prime}(s, t)$ for any other $(s, t) \in I(S,<)$. Then $\Sigma \cup\left\{I(,),\left(\lambda^{\prime}\right)\right\}$ is consistent. 
Proof. Almost immediate from Axiom (I2).

\section{Lemma 2.5.8.}

$$
\vdash I(n, x, y)\left(\left\{\bar{A}_{i j}\right\}\right) \rightarrow I(n, x, y)\left(\left\{\bar{A}_{i j}^{\prime}\right\}\right) \vee I(n, x, y)\left(\left\{\bar{A}_{i j}^{\prime \prime}\right\}\right),
$$

where $\bar{A}_{i_{1} j_{1}}^{\prime}=\bar{B} \wedge \bar{A}_{i_{1} j_{1}}$ and $\bar{A}_{i_{1} j_{1}}^{\prime \prime}=\neg \bar{B} \wedge \bar{A}_{i_{1} j_{1}}$ for some wff $\bar{B}$ and some interval $\left(i_{1}, j_{1}\right)$, while $\bar{A}_{i j}=\bar{A}_{i j}^{\prime}=\bar{A}_{i j}^{\prime \prime}$ for any other $(i, j)$.

Proof. By PC,

$$
\vdash \bar{A}_{i_{1} j_{1}} \rightarrow\left(\bar{B} \wedge \bar{A}_{i_{1} j_{1}}\right) \vee\left(\neg \bar{B} \wedge \bar{A}_{i_{1} j_{1}}\right) .
$$

By taking the contraposition of (1),

$$
\vdash \neg\left(\bar{B} \wedge \bar{A}_{i_{1} j_{1}}\right) \wedge \neg\left(\neg \bar{B} \wedge \bar{A}_{i_{1} j_{1}}\right) \rightarrow \neg \bar{A}_{i_{1} j_{1}} .
$$

From (2) by Axiom (I1) and Rule (RIN),

$$
\vdash I e(n, x, y)\left(\left\{\neg \bar{A}_{i j}^{\prime}\right\}\right) \wedge I e(n, x, y)\left(\left\{\neg \bar{A}_{i j}^{\prime \prime}\right\}\right) \rightarrow I e(n, x, y)\left(\left\{\neg \bar{A}_{i j}\right\}\right) .
$$

By taking the contraposition of (3),

$$
\vdash \bar{B} \rightarrow \bar{B}^{\prime} \vee \bar{B}^{\prime \prime},
$$

where

$$
\begin{aligned}
& \bar{B}=\neg I e(n, x, y)\left(\left\{\neg \bar{A}_{i j}\right\}\right), \bar{B}^{\prime}=\neg I e(n, x, y)\left(\left\{\neg \bar{A}_{i j}^{\prime}\right\}\right), \quad \text { and } \\
& \bar{B}^{\prime \prime}=\neg I e(n, x, y)\left(\left\{\neg A_{i j}^{\prime \prime}\right\}\right) .
\end{aligned}
$$

It is easy to see by the definition of $\operatorname{Ie}(n, 1, r)$ and Rule (RID) that:

$$
\begin{aligned}
& \vdash \neg I e(n, x, y)\left(\left\{\neg \bar{A}_{i j}\right\}\right) \leftrightarrow I(n, x, y)\left(\left\{\bar{A}_{i j}\right\}\right) . \\
& \vdash \neg I e(n, x, y)\left(\left\{\neg \bar{A}_{i j}^{\prime}\right\}\right) \leftrightarrow I(n, x, y)\left(\left\{\bar{A}_{i j}^{\prime}\right\}\right) . \\
& \vdash \neg I e(n, x, y)\left(\left\{\neg \bar{A}_{i j}^{\prime \prime}\right\}\right) \leftrightarrow I(n, x, y)\left(\left\{\bar{A}_{i j}^{\prime \prime}\right\}\right) .
\end{aligned}
$$

From (4)-(7), we can get the desired result.

Corollary 2.5.9. Suppose that:

(a) $\bar{\Sigma}$ is a theory.

(b) $\lambda=(S,<,(\bar{s}, \bar{t}), \Psi)$ is a finite TDI-sequence.

(c) $\bar{B}$ is a wff.

(d) $\bar{\Sigma} \cup\{I(,),(\lambda)\}$ is consistent.

We define TDI-sequences $\lambda^{\prime}=\left(S,<,(s, t), \Psi^{\prime}\right)$ and $\lambda^{\prime \prime}=\left(S,<,(\bar{s}, \bar{t}), \Psi^{\prime \prime}\right)$, where $\Psi^{\prime}\left(s_{1}, t_{1}\right)=\{\bar{B}\} \cup \Psi\left(s_{1}, t_{1}\right)$ and $\Psi^{\prime \prime}\left(s_{1}, t_{1}\right)=\{\neg \bar{B}\} \cup \Psi\left(s_{1}, t_{1}\right)$ for some interval $\left(s_{1}, t_{1}\right)$, while $\Psi(s, t)=\Psi^{\prime}(s, t)=\Psi^{\prime \prime}(s, t)$ for any other $(s, t)$. Then we 
can conclude that at least one of $\bar{\Sigma} \cup\left\{I(n, x, y)\left(\lambda^{\prime}\right)\right\}$ and $\bar{\Sigma} \cup\left\{I(n, x, y)\left(\lambda^{\prime \prime}\right)\right\}$ is consistent.

Lemma 2.5.10. Suppose that:

(a) $\bar{\Sigma}$ is a theory.

(b) $\lambda=(S,<,(\bar{s}, \bar{t}), \Psi)$ is a finite TDI-sequence.

(c) $\bar{\Sigma} \cup\{I(,),(\lambda)\}$ is consistent.

(d) For some $\left(s_{1}, t_{1}\right) \in I(S,<)$ and some finite TDI-sequence $\lambda^{\prime}$, $I(,),\left(\lambda^{\prime}\right) \in \Psi\left(s_{1}, t_{1}\right)$.

Then there exists such a finite TDI-sequence $\lambda^{\prime \prime}$ that:

(a) $\bar{\Sigma} \cup\left\{I(,),\left(\lambda^{\prime \prime}\right)\right\}$ is consistent.

(b) $\lambda \subseteq \lambda^{\prime \prime}$.

(c) $\lambda^{\prime \prime}$ is a sum of $\lambda$ and $\lambda^{\prime}$ with respect to $\left(s_{1}, t_{1}\right)$.

Proof. Almost immediate from Axiom (I4).

Lemma 2.5.11. If $I(n, x, y)\left(\left\{\bar{A}_{i j}\right\}\right)$ is consistent, then each $\bar{A}_{i j}$ is consistent.

Proof. Suppose, for the sake of contradiction, that for some $\left(i_{1}, j_{1}\right)$,

Then by Rule (RIN),

$$
\vdash \neg A_{i_{1} j_{1}}
$$

$$
\vdash \operatorname{Ie}(n, x, y)\left(\left\{\neg \bar{A}_{i j}\right\}\right) \text {. }
$$

It is easy to see, by the definition of $\operatorname{Ie}(n, 1, r)$ and Rule (RID), that:

$$
\vdash I e(n, x, y)\left(\left\{\neg \bar{A}_{i j}\right\}\right) \longleftrightarrow \neg I(n, x, y)\left(\left\{\bar{A}_{i j}\right\}\right) .
$$

From (2) and (3),

$$
\vdash \neg I(n, x, y)\left(\left\{\bar{A}_{i j}\right\}\right)
$$

which is a contradiction.

Corollary 2.5.12. Let $\lambda=(S,<,(\bar{s}, \bar{t}), \Psi)$ be a finite TDI-sequence such that $I(,),(\lambda)$ is consistent. Then $\Psi(s, t)$ is consistent for any $(s, t) \in I(S,<)$.

Lemma 2.5.13. Suppose that:

(a) $\lambda=(S,<,(\bar{s}, \bar{t}), \Psi)$ is a finite TDI-sequence.

(b) $I(,),(\lambda)$ is consistent.

(c) $\neg I(,),\left(\alpha^{\prime}\right) \in \Psi\left(s_{1}, t_{1}\right)$ for some $\left(s_{1}, t_{1}\right) \in I(S,<)$ and some DIsequence $\alpha^{\prime}=\left(S^{\prime},<,\left(\bar{s}^{\prime}, \bar{t}^{\prime}\right), \Omega^{\prime}\right)$. 
(d) $f$ is an order monomorphism from $\left(S^{\prime},<\right)$ to $(S,<)$ such that $f\left(\bar{s}^{\prime}\right)$ $=s_{1}$ and $f\left(\bar{t}^{\prime}\right)=t_{1}$.

Then we can conclude that $\Omega^{\prime}(s, t) \notin \Psi(f(s), f(t))$ for some $(s, t) \in I\left(S^{\prime},<\right)$.

Proof. This follows mainly from Axiom (I5). The details are left to the reader.

Since WFF is a countable set, we can enumerate all wffs in the following manner:

$$
\bar{A}_{1}, \bar{A}_{2}, \bar{A}_{3}, \bar{A}_{4}, \bar{A}_{5}, \ldots, \bar{A}_{i}, \ldots
$$

In the rest of this section we fix such an enumeration. We also need an auxiliary countable set $V$, whose elements are:

$$
v_{1}, v_{2}, v_{3}, v_{4}, v_{5}, \ldots, v_{i}, \ldots
$$

In the rest of this section we fix such $V$.

Now, with these preparations, we can present the proof of Theorem 2.5.2. Let $\bar{\Sigma}_{0}$ be the given consistent theory. It is sufficient to construct a TI-model realizing this theory. The outline of the proof goes as follows:

(a) We construct a finite TDI-sequence $\lambda_{n}=\left(S_{n},<,\left(v_{1}, v_{2}\right), \Psi_{n}\right)$ at each stage $n$ inductively such that:

(i) $S_{n} \subseteq V$

(ii) $\bar{\Sigma}_{0} \cup\left\{I(,),\left(\lambda_{n}\right)\right\}$ is consistent.

(iii) $\lambda_{n} \subseteq \lambda_{(n+1)}$ for any $n$.

(b) We can get a desired TI-model as the limit of $\lambda_{n}$ 's.

To explain the detailed construction of $\lambda_{n}$ 's, we need to classify all stages $n$ into 5 cases. Given a natural number $n$ and a prime number $p$, we denote by $E(p, n)$ one plus the exponent of $p$ in the prime decomposition of $n$. For example, $E(2,8)=4, E(5,100)=3$, etc. Then the detailed construction of $\lambda$ $=\left(S_{n},<,\left(v_{1}, v_{2}\right), \Psi_{n}\right)$ goes as follows:

\section{Case 1:}

$\lambda_{1}=\left(S_{1},<,\left(v_{1}, v_{2}\right), \Psi_{1}\right)$, where
(a) $S_{1}=\left\{v_{1}, v_{2}\right\}$.
(b) $v_{1}<v_{2}$.
(c) $\Psi\left(v_{1}, v_{2}\right)=\emptyset$ (the empty). 
It is easy to see, by Axiom (I0), that $\bar{\Sigma}_{0} \cup\left\{I(,),\left(\lambda_{1}\right)\right\}$ is consistent.

Case 2:

Suppose $n$ is such that:

(a) $E(2, n)=2$.

(b) $\bar{A}_{E(3, n)} \in \bar{\Sigma}_{0}$.

Then $\lambda_{n}=\left(S_{n},<,\left(v_{1}, v_{2}\right), \Psi_{n}\right)$ shall be such that:

(a) $\left(S_{n},<\right)=\left(S_{(n-1)},<\right)$.

(b) $\Psi_{n}\left(v_{1}, v_{2}\right)=\left\{\bar{A}_{E(3, n)}\right\} \cup \Psi_{(n-1)}\left(v_{1}, v_{2}\right)$, while $\Psi_{n}(s, t)=\Psi_{(n-1)}(s, t)$ for any other $(s, t) \in I\left(S_{n},<\right)$.

Then obviously $\lambda_{(n-1)} \subseteq \lambda_{n}$. By Lemma 2.5 .7 , it is easy to see that $\bar{\Sigma}_{0} U$ $\left\{I(,),\left(\lambda_{n}\right)\right\}$ is consistent provided $\bar{\Sigma}_{0} \cup\left\{I(,),\left(\lambda_{(n-1)}\right)\right\}$ is consistent.

Case 3:

Suppose $n$ is such that:

(a) $E(2, n)=3$.

(b) $\left(v_{E(5, n)}, v_{E(7, n)}\right) \in I\left(S_{(n-1)},<\right)$.

$\lambda^{\prime}=\left(S_{(n-1)},<,\left(v_{1}, v_{2}\right), \Psi^{\prime}\right)$ and $\lambda^{\prime \prime}=\left(S_{(n-1)},<,\left(v_{1}, v_{2}\right), \Psi^{\prime \prime}\right)$ shall be such that:

(a) $\Psi^{\prime}\left(s_{1}, t_{1}\right)=\left\{\bar{A}_{E(3, n)}\right\} \cup \Psi_{(n-1)}\left(s_{1}, t_{1}\right)$, while $\Psi^{\prime \prime}\left(s_{1}, t_{1}\right)=\left\{\neg \bar{A}_{E(3, n)}\right\}$ $\cup \Psi_{(n-1)}\left(s_{1}, t_{1}\right)$, where $s_{1}=v_{E(5, n)}$ and $t_{1}=v_{E(7, n)}$.

(b) $\Psi_{(n-1)}(s, t)=\Psi^{\prime}(s, t)=\Psi^{\prime \prime}(s, t)$ for any other $(s, t) \in I\left(S_{(n-1)},<\right)$.

Then obviously $\lambda_{(n-1)} \subseteq \lambda^{\prime}$ and $\lambda_{(n-1)} \subseteq \lambda^{\prime \prime}$. By Corollary 2.5.9, it is easy to see that at least one of $\bar{\Sigma}_{0} \cup\left\{I(,),\left(\lambda^{\prime}\right)\right\}$ and $\bar{\Sigma}_{0} \cup\left\{I(,),\left(\lambda^{\prime \prime}\right)\right\}$ is consistent provided $\bar{\Sigma}_{0} \cup\left\{I(,),\left(\lambda_{(n-1)}\right)\right\}$ is consistent. $\lambda_{n}$ shall be such one of $\lambda^{\prime}$ and $\lambda^{\prime \prime}$.

Case 4:

Suppose $n$ is such that:

(a) $E(2, n)=4$.

(b) $\left(v_{E(5, n)}, v_{E(7, n)}\right) \in I\left(S_{(n-1)},<\right)$.

(c) $A_{E(3, n)} \in \Psi_{(n-1)}\left(v_{E(5, n)}, v_{E(7, n)}\right)$.

(d) $A_{E(3, n)}$ is of the form $I(,),\left(\lambda^{\prime}\right)$ for some TDI-sequence $\lambda^{\prime}$.

By Lemma 2.5.10, it is easy to see that there exists a TDI-sequence $\lambda^{\prime \prime}$ $=\left(S^{\prime \prime},<,\left(v_{1}, v_{2}\right), \Psi^{\prime \prime}\right)$ such that: 
(a) $S^{\prime \prime} \subseteq V$.

(b) $\lambda_{(n-1)} \subseteq \lambda^{\prime \prime}$.

(c) $\lambda^{\prime \prime}$ is a sum of $\lambda_{(n-1)}$ and $\lambda^{\prime}$ with respect to $\left(v_{E(5, n)}, v_{E(7, n)}\right)$.

(d) $\bar{\Sigma}_{0} \cup\left\{I(,),\left(\lambda^{\prime \prime}\right)\right\}$ is consistent provided $\bar{\Sigma}_{0} \cup\left\{I(,),\left(\lambda_{(n-1)}\right)\right\}$ is consistent.

$\lambda_{n}$ shall be such $\lambda^{\prime \prime}$.

Case 5:

Otherwise $\lambda_{n}=\lambda_{(n-1)}$.

The following lemmas follow directly from the manner of the construction of $\lambda_{n}{ }^{9} \mathrm{~s}$.

Lemma 2.5.14. $\lambda_{1} \subseteq \lambda_{2} \subseteq \lambda_{3} \subseteq \lambda_{4} \subseteq \cdots \subseteq \lambda_{n} \subseteq \cdots$.

Lemma 2.5.15. $I(,),\left(\lambda_{n}\right)$ is consistent for any $n$.

Thus we can define a TDI-sequence $\lambda_{\infty}=\left(S_{\infty},<,\left(v_{1}, v_{2}\right), \Psi_{\infty}\right)$ as the limit of $\lambda_{n}$ 's. Specifically,

(a) $\left(S_{\infty},<\right)=\cup_{n \geq 1}\left(S_{n},<\right)$.

(b) $\Psi_{\infty}(s, t)=\cup_{n} \Psi_{n}(s, t)$ for any $(s, t) \in I\left(S_{\infty},<\right)$.

Lemma 2.5.16. $\bar{\Sigma}_{0} \subseteq \Psi_{\infty}\left(v_{1}, v_{2}\right)$.

Proof. Immediate from the construction of $\lambda_{n}$ 's of Case 2.

Lemma 2.5.17. $\Psi_{\infty}(s, t)$ is maximal consistent for any $(s, t) \in I\left(S_{\infty},<\right)$.

Proof. Almost immediate from the construction of $\lambda_{n}$ 's of Case 3, Lemma 2.5.12, and Lemma 2.5.15.

Corollary 2.5.18. For any $(s, t) \in I\left(S_{\infty},<\right)$,

(1) $\neg \bar{A} \in \Psi_{\infty}(s, t)$ iff $\bar{A} \notin \Psi_{\infty}(s, t)$.

(2) $(\bar{A} \rightarrow \bar{B}) \in \Psi_{\infty}(s, t)$ iff $\bar{A} \notin \Psi_{\infty}(s, t)$ or $\bar{B} \in \Psi_{\infty}(s, t)$.

Lemma 2.5.19. I $(n, x, y)\left(\left\{\bar{A}_{i j}\right\}\right) \in \Psi_{\infty}(s, t)$ iff for some $s_{i}$ 's $(1 \leq i \leq n)$ of $S_{\infty}$, $s_{1}<\cdots<s_{n}, s_{x}=s, s_{y}=t$ and $\bar{A}_{i j} \in \Psi\left(s_{i}, s_{j}\right)$ for any $(i, j)$.

Proof. Only if part: Immediate from the construction of $\lambda_{n}$ 's of Case 4. If part: Suppose, for the sake of contradiction, that $I(n, x, y)\left(\left\{\bar{A}_{i j}\right\}\right) \notin \Psi(s, t)$. Then by Lemma 2.5.17 $\neg I(n, x, y)\left(\left\{\bar{A}_{i j}\right\}\right) \in \Psi_{\infty}(s, t)$. We can conclude, by Lemma 2.5.13, that for any $s_{i}$ 's $(1 \leq i \leq n)$ such that $s_{1}<\cdots<s_{n}, s_{x}=s$ and $s_{y}=t$, $\bar{A}_{i j} \notin \Psi_{\infty}\left(s_{i}, s_{j}\right)$ for some $(i, j)$. This is a contradiction. 
We define $D_{\infty}$ as follows:

For any $\bar{p} \in \bar{P}$ and any $(s, t) \in I\left(S_{\infty},<\right)$,

$$
D_{\infty}(\bar{p}, s, t)=1 \text { iff } \bar{p} \in \Psi_{\infty}(s, t) .
$$

We will show that $\left(S_{\infty},<, D_{\infty},\left(v_{1}, v_{2}\right)\right)$ is indeed a desired TI-model.

Proposition 2.5.20. In the TI-structure $\left(S_{\infty},<, D_{\infty}\right)$, for any wff $\bar{A}$ and $(s, t) \in I\left(S_{\infty},<\right),\|\bar{A}\|_{(s, t)}=1$ iff $\bar{A} \in \Psi_{\infty}(s, t)$.

Proof. We can prove this by induction on the construction of wffs.

(a) $\bar{A}=\bar{p}$ : Immediate from the definition of $D_{\infty}$.

(b) $\bar{A}=\neg \bar{B}:$ Immediate from Corollary 2.5.18.

(c) $\bar{A}=\bar{B} \rightarrow \bar{C}:$ Immediate from Corollary 2.5.18.

(d) $\bar{A}=I(n, 1, r)\left(\left\{\bar{A}_{i j}\right\}\right)$ : Immediate from Lemma 2.5.19.

Corollary 2.5.21. In the TI-structure $\left(S_{\infty},<, D_{\infty}\right),\|\bar{A}\|_{\left(v_{1}, v_{2}\right)}=1$ for any $\bar{A} \in \bar{\Sigma}_{0} . \quad$ That is to say, $\left(S_{\infty},<, D_{\infty},\left(v_{1}, v_{2}\right)\right) \models \bar{\Sigma}_{0}$.

Proof. Immediate from Lemma 2.5.16 and Proposition 2.5.20.

This completes the proof of Theorem 2.5.2.

\section{Chapter 3}

\section{The Propositional Logic IM}

\section{§3.1. Formal Language LIM}

Definition 3.1.1. Our formal language LIM consists of the following symbols:

(a) a countable set $P$ of s-variables ("s" for "state"): $p, q, p^{\prime}, q^{\prime}, \ldots$;

(b) a countable set $\bar{P}$ of p-variables ("p" for "process"): $\bar{p}, \bar{q}, \bar{p}^{\prime}, \bar{q}^{\prime}, \ldots$;

(c) classical connectives: $\neg, \rightarrow$;

(d) tense operators $\bar{M}$ 's: $\bar{M}(n, z)$, where $n$ and $z$ range over all natural numbers such that $1 \leq z \leq n$;

(e) tense operators $\bar{I}^{\prime}$ : : $\bar{I}(n, x, y)$, where $n, x$ and $y$ range over all natural numbers such that $1 \leq x<y \leq n$;

(f) parentheses: $($,$) .$

Definition 3.1.2. We define the notions of a M-wff ("M" for "moment") 
and an I-wff ("I" for "interval") by induction as follows:

(a1) Any s-variable $p$ is an M-wff.

(a2) Any p-variable $\bar{p}$ is an I-wff.

(b1) If $A$ and $B$ are M-wffs, so too are $(\neg A)$ and $(A \rightarrow B)$.

(b2) If $\bar{A}$ and $\bar{B}$ are I-wffs, so too are $(\neg \bar{A})$ and $(\bar{A} \rightarrow \bar{B})$.

(c1) If for any $1 \leq i \leq n, A_{i}$ is an M-wff and for any $1 \leq i<j \leq n, \bar{B}_{i j}$ is an I-wff, then $\bar{M}(n, z)\left(A_{1}, \ldots, A_{n}, \bar{B}_{12}, \ldots, \bar{B}_{(n-1) n}\right)$ is a M-wff, where $A_{i}$ 's and $B_{i j}$ 's are arranged in lexicographical order with respect to $i$ or $(i, j)$.

(c2) If for any $1 \leq i<j \leq n, \bar{A}_{i j}$ is an I-wff and for any $1 \leq i \leq n, B_{i}$ is an M-wff, then $\bar{I}(n, x, y)\left(\bar{A}_{12}, \ldots, \bar{A}_{(n-1) n}, B_{1}, \ldots, B_{n}\right)$ is an I-wff, where $\bar{A}_{i j}$ 's and $B_{i}$ 's are arranged in lexicographical order with respect to $(i, j)$ or $i$.

First of all, our usage of parentheses is very loose in this chapter as in the previous chapter. We should notice that the arity of $\bar{M}(n, z)$ is $n+\frac{n(n-1)}{2}$ $=\frac{n(n+1)}{2}$. Similarly the arity of $\bar{I}(n, x, y)$ is $\frac{n(n-1)}{2}+n=\frac{n(n+1)}{2}$. For the sake of notational simplicity, we will often write $\bar{M}(n, z)\left(\left\{A_{i}\right\}_{1 \leq i \leq n},\left\{\bar{B}_{i j}\right\}_{1 \leq i<j \leq n}\right)$ or $\bar{M}(n, z)\left(\left\{A_{i}\right\},\left\{\bar{B}_{i j}\right\}\right)$ rather than $\bar{M}(n, z)\left(A_{1}, \ldots, A_{n}, \bar{B}_{12}, \ldots, \bar{B}_{(n-1) n}\right)$. Similarly for $\bar{I}(n, x, y)\left(\left\{\bar{A}_{i j}\right\}_{1 \leq i<j \leq n},\left\{B_{i}\right\}_{1 \leq i \leq n}\right)$ or $\bar{I}(n, x, y)\left(\left\{\bar{A}_{i j}\right\},\left\{B_{i}\right\}\right)$. We will make use of $\bar{M} e(n, z)\left(\left\{A_{i}\right\},\left\{\bar{B}_{i j}\right\}\right)$ and $\bar{I} e(n, x, y)\left(\left\{\bar{A}_{i j}\right\},\left\{B_{i}\right\}\right)$ as abbreviations of $\neg \bar{M}(n, z)\left(\left\{\neg A_{i}\right\},\left\{\neg \bar{B}_{i j}\right\}\right)$ and $\neg \bar{I}(n, x, y)\left(\left\{\neg \bar{A}_{i j}\right\},\left\{\neg B_{i}\right\}\right)$ respectively. Symbols for conjunction $(\wedge)$, disjunction $(\vee)$ and material equivalence $(\leftrightarrow)$ are defined as usual. T Tand $F$ are defined as abbreviations of $(p \rightarrow p)$ and $\neg(p \rightarrow p)$ for some s-variable $p$ respectively. $\bar{T}$ and $\bar{F}$ are defined as abbreviations of $(\bar{p} \rightarrow \bar{p})$ and $\neg(\bar{p} \rightarrow \bar{p})$ for some p-variable $\bar{p}$ respectively. We will use $A, B, A_{i j}$, etc. for M-wffs and $\bar{A}, \bar{B}, \bar{A}_{i j}$, etc. for I-wffs. The least confusion between M-wffs and I-wffs would cause the greatest perplexity to the reader in this chapter.

\section{§3.2. Semantics for IM}

Definition 3.2.1. A TIM-structure is a 4-tuple $(S,<, d, D)$, where

(a) $S$ is a set (called the "time").

(b) $<$ is a strict linear order in $S$.

(c) $d$ is a function from $P \times S$ to $\{0,1\}$. That is, $d$ assigns a truth-value to each s-variable at each moment. 
(d) $D$ is a function from $\bar{P} \times I(S,<)$ to $\{0,1\}$. That is, $D$ assigns a truth-value to each p-variable at each interval.

Definition 3.2.2. Given a TlM-structure $(S,\langle, d, D)$, the truth-value $\|A\|_{t}$ of an M-wff $A$ at a moment $t$ and the truth-value $\|\bar{A}\|_{(s, t)}$ of an I-wff $\bar{A}$ at an interval $(s, t)$ are defined by induction as follows:

(a1) $\|p\|_{t}=d(p, t)$, where $p$ is a s-variable.

(a2) $\|\bar{p}\|_{(s, t)}=D(\bar{p}, s, t)$, where $p$ is a p-variable.

(b1) $\|\neg A\|_{t}=1$ iff $\|A\|_{t}=0$.

(b2) $\|\neg \bar{A}\|_{(s, t)}=1$ iff $\|\bar{A}\|_{(s, t)}=0$.

(c1) $\|A \rightarrow B\|_{t}=1$ iff $\|A\|_{t}=0$ or $\|B\|_{t}=1$.

(c2) $\|\bar{A} \rightarrow \bar{B}\|_{(s, t)}=1$ iff $\|\bar{A}\|_{(s, t)}=0$ or $\|\bar{B}\|_{(s, t)}=1$.

(d1) $\left\|\bar{M}(n, z)\left(\left\{A_{i}\right\},\left\{\bar{B}_{i j}\right\}\right)\right\|_{t}=1$ iff for some $s_{i}$ 's $(1 \leq i \leq n), s_{1}<\cdots<s_{n}$, $s_{z}=t,\left\|A_{i}\right\|_{s_{i}}=1$ for any $i$ and $\left\|\bar{B}_{i j}\right\|_{\left(s_{i}, s_{j}\right)}=1$ for any $(i, j)$.

(d2) $\left\|\bar{I}(n, x, y)\left(\left\{\bar{A}_{i j}\right\},\left\{B_{i}\right\}\right)\right\|_{(s, t)}=1$ iff for some $s_{i}$ 's $(1 \leq i \leq n), s_{1}<\cdots<s_{n}$, $s_{x}=s, s_{y}=t,\left\|\bar{A}_{i j}\right\|_{\left(s_{i}, s_{j}\right)}=1$ for any $(i, j)$ and $\left\|B_{i}\right\|_{s_{i}}=1$ for any $i$.

By way of example, the situations depicted in Figures 4-9 are represented as follows:

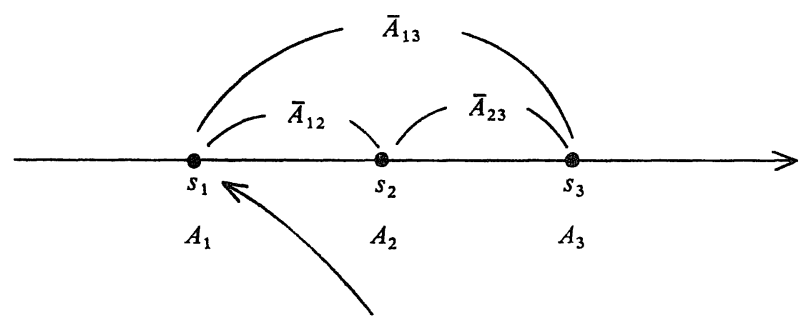

the distinguished reference moment

Figure 4.

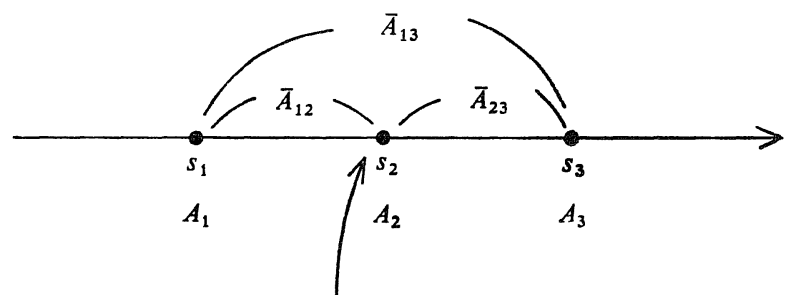

the distinguished reference moment

Figure 5. 


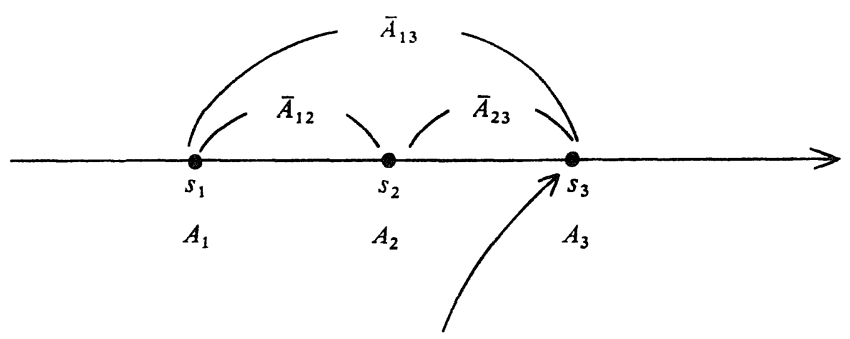

the distinguished

reference moment

Figure 6.

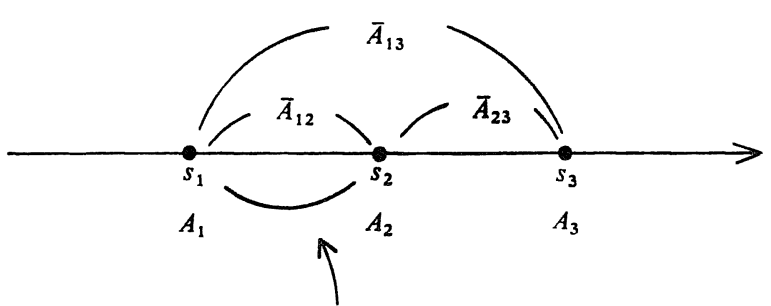

the distinguished reference interval

Figure 7.

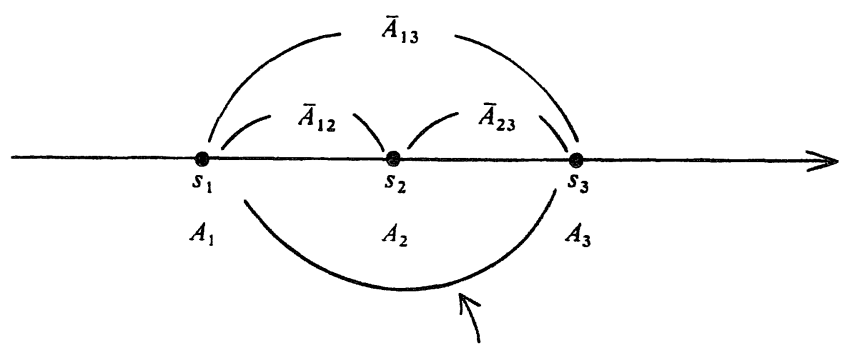

the distinguished reference interval

Figure 8.

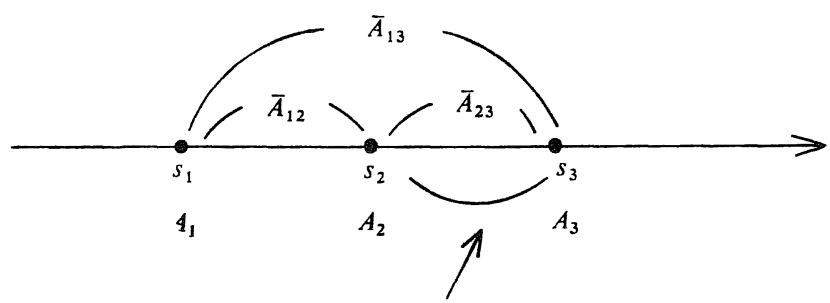

the distinguished reference interval

Figure 9. 
(1) $\bar{M}(3,1)\left(A_{1}, A_{2}, A_{3}, \bar{A}_{12}, \bar{A}_{13}, \bar{A}_{23}\right)$.

(2) $\bar{M}(3,2)\left(A_{1}, A_{2}, A_{3}, \bar{A}_{12}, \bar{A}_{13}, \bar{A}_{23}\right)$.

(3) $\bar{M}(3,3)\left(A_{1}, A_{2}, A_{3}, \bar{A}_{12}, \bar{A}_{13}, \bar{A}_{23}\right)$.

(4) $\bar{I}(3,1,2)\left(\bar{A}_{12}, \bar{A}_{13}, \bar{A}_{23}, A_{1}, A_{2}, A_{3}\right)$.

(5) $\bar{I}(3,1,3)\left(\bar{A}_{12}, \bar{A}_{13}, \bar{A}_{23}, A_{1}, A_{2}, A_{3}\right)$.

(6) $\bar{I}(3,2,3)\left(\bar{A}_{12}, \bar{A}_{13}, \bar{A}_{23}, A_{1}, A_{2}, A_{3}\right)$ 。

It is important to notice that $\|A\|_{(s, t)}$ and $\|\bar{A}\|_{t}$ are undefined at all. With the above definition, it is easy to see that:

(e1) $\left\|\bar{M} e(n, z)\left(\left\{A_{i}\right\},\left\{\bar{B}_{i j}\right\}\right)\right\|_{t}=1$ iff for any $s_{i}$ 's $(1 \leq i \leq n)$ such that $s_{1}<\cdots$ $<s_{n}$ and $s_{z}=t,\left\|A_{i}\right\|_{s_{i}}=1$ for some $i$ or $\left\|\bar{B}_{i j}\right\|_{\left(s_{i}, s_{j}\right)}=1$ for some $(i, j)$.

(e2) $\left\|\bar{I} e(n, x, y)\left(\left\{\bar{A}_{i j}\right\},\left\{B_{i}\right\}\right)\right\|_{(s, t)}=1$ iff for any $s_{i}$ 's $(1 \leq i \leq n)$ such that $s_{1}<\cdots<s_{n}, s_{x}=s$ and $s_{y}=t,\left\|\bar{A}_{i j}\right\|_{\left(s_{i}, s_{j}\right)}=1$ for some $(i, j)$ or $\left\|B_{i}\right\|_{s_{i}}=1$ for some $i$.

\section{Definition 3.2.3.}

(1) A TMI-model is a 5-tuple $\rho=(S,<, d, D, \bar{s})$, where

(a) $(S,<, d, D)$ is a TIM-structure.

(b) $\bar{s} \in S$ (the present moment).

(2) A TIM-model is a 5-tuple $\mu=(S,<, d, D,(\bar{s}, \bar{t}))$, where

(a) $(S,<, d, D)$ is a TIM-structure.

(b) $(\bar{s}, \bar{t}) \in I(S,<)$ (the present interval).

A set of M-wffs is called an M-theory. A set of I-wffs is called an I-theory. Definition 3.2.4.a.

(1) A TMI-model $\rho=(S,<, d, D, \bar{s})$ is said to realize an M-wff $A$, notation: $\rho \vDash A$, if $\|A\|_{\bar{s}}=1$.

(2) A TMI-model $\rho=(S,<, d, D, \bar{s})$ is said to realize an M-theory $\Sigma$, notation: $\rho \vDash \Sigma$, if $\|A\|_{\bar{s}}=1$ for any $A \in \Sigma$.

(3) An M-wff $A$ is said to be realizable if for some TMI-model $\rho, \rho \vDash A$.

(4) An M-theory $\Sigma$ is said to be realizable if for some TMI-model $\rho, \rho \vDash \Sigma$.

(5) An M-wff $A$ is said to be valid, notation: $\vDash A$, if $\rho \vDash A$ for any TMI-model $\rho$. Definition 3.2.4.b.

(1) A TIM-model $\mu=(S,<, d, D,(\bar{s}, \bar{t}))$ is said to realize an I-wff $\bar{A}$, notation: $\mu \models \bar{A}$, if $\|\bar{A}\|_{(\bar{s}, \bar{t})}=1$.

(2) A TIM-model $\mu=(S,<, d, D,(\bar{s}, \bar{t}))$ is said to realize an I-theory $\bar{\Sigma}$, 
notation: $\mu \models \bar{\Sigma}$, if $\|\bar{A}\|_{(\bar{s}, \bar{t})}=1$ for any $\bar{A} \in \bar{\Sigma}$.

(3) An I-wff $\bar{A}$ is said to be realizable if for some TIM-model $\mu, \mu \vDash \bar{A}$.

(4) An I-theory $\bar{\Sigma}$ is said to be realizable if for some TIM-model $\mu, \mu \models \bar{\Sigma}$.

(5) An I-wff $\bar{A}$ is said to be valid, notation: $\vDash \bar{A}$, if $\mu \models \bar{A}$ for any TIM-model $\mu$.

The rest of this section is devoted to some examples of valid wffs.

Example 3.2.5.a. The following $M-w f f$ is valid.

$$
\begin{aligned}
& \bar{M}(4,3)\left(A_{1}, A_{2}, A_{3}, A_{4}, \bar{A}_{12}, \bar{A}_{13}, \bar{A}_{14}, \bar{A}_{23}, \bar{A}_{24}, \bar{A}_{34}\right) \longrightarrow \\
& \bar{M}(3,3)\left(A_{1}, A_{2}, A_{3}, \bar{A}_{12}, \bar{A}_{13}, \bar{A}_{23}\right) .
\end{aligned}
$$

Example 3.2.5.b. The following I-wff is valid.

$$
\begin{aligned}
& \bar{I}(4,2,3)\left(\bar{A}_{12}, \bar{A}_{13}, \bar{A}_{14}, \bar{A}_{23}, \bar{A}_{24}, \bar{A}_{34}, A_{1}, A_{2}, A_{3}, A_{4}\right) \longrightarrow \\
& \tilde{I}(3,2,3)\left(\bar{A}_{12}, \bar{A}_{13}, \bar{A}_{23}, A_{1}, A_{2}, A_{3}\right) .
\end{aligned}
$$

Example 3.2.6.a. The following $M-w f f$ is valid.

$$
\bar{M}(3,2)\left(A_{1}, A_{2}, A_{3}, \bar{A}_{12}, \bar{A}_{13}, \bar{A}_{23}\right) \longrightarrow C_{1} \vee C_{2} \vee C_{3},
$$

where

(a) $\bar{A}_{23}=\bar{I}(3,2,3)\left(\bar{A}_{12}^{\prime}, \bar{A}_{13}^{\prime}, \bar{A}_{23}^{\prime}, A_{1}^{\prime}, A_{2}^{\prime}, A_{3}^{\prime}\right)$.

(b) $C_{1}=\bar{M}(4,3)\left(A_{1}, A_{1}^{\prime}, A_{2} \wedge A_{2}^{\prime}, A_{3} \wedge A_{3}^{\prime}, \bar{T}, \bar{A}_{12}, \bar{A}_{13}, \bar{A}_{12}^{\prime}, \bar{A}_{13}^{\prime}\right.$, $\left.\bar{A}_{23} \wedge \bar{A}_{23}^{\prime}\right)$.

(c) $C_{2}=\bar{M}(3,2)\left(A_{1} \wedge A_{1}^{\prime}, A_{2} \wedge A_{2}^{\prime}, A_{3} \wedge A_{3}^{\prime}, \bar{A}_{12} \wedge \bar{A}_{12}^{\prime}, \bar{A}_{13} \wedge \bar{A}_{13}^{\prime}\right.$, $\left.\bar{A}_{23} \wedge \bar{A}_{23}^{\prime}\right)$.

(d) $C_{3}=\bar{M}(4,3)\left(A_{1}^{\prime}, A_{1}, A_{2} \wedge A_{2}^{\prime}, A_{3} \wedge A_{3}^{\prime}, \bar{T}, \bar{A}_{12}^{\prime}, \bar{A}_{13}^{\prime}, \bar{A}_{12}, \bar{A}_{13}\right.$, $\left.\bar{A}_{23} \wedge \bar{A}_{23}^{\prime}\right)$.

Example 3.2.6.b. The following I-wff is valid.

$$
\bar{I}(3,1,2)\left(\bar{A}_{12}, \bar{A}_{13}, \bar{A}_{23}, A_{1}, A_{2}, A_{3}\right) \longrightarrow \bar{C}_{1} \vee \bar{C}_{2} \vee \bar{C}_{3},
$$

where

(a) $\quad \bar{A}_{23}=\bar{I}(3,2,3)\left(\bar{A}_{12}^{\prime}, \bar{A}_{13}^{\prime}, \bar{A}_{23}^{\prime}, A_{1}^{\prime}, A_{2}^{\prime}, A_{3}^{\prime}\right)$.

(b) $\quad \bar{C}_{1}=\bar{I}(4,1,3)\left(\bar{T}, \bar{A}_{12}, \bar{A}_{13}, \bar{A}_{12}^{\prime}, \bar{A}_{13}^{\prime}, \bar{A}_{23} \wedge \bar{A}_{23}^{\prime}, A_{1}, A_{1}^{\prime}, A_{2} \wedge A_{2}^{\prime}\right.$, $\left.A_{3} \wedge A_{3}^{\prime}\right)$.

(c) $\bar{C}_{2}=\bar{I}(3,1,2)\left(\bar{A}_{12} \wedge \bar{A}_{12}^{\prime}, \bar{A}_{13} \wedge \bar{A}_{13}^{\prime}, \bar{A}_{23} \wedge \bar{A}_{23}^{\prime}, A_{1} \wedge A_{1}^{\prime}, A_{2} \wedge A_{2}^{\prime}\right.$, $\left.A_{3} \wedge A_{3}^{\prime}\right)$.

(d) $\bar{C}_{3}=\bar{I}(4,1,3)\left(\bar{T}, \bar{A}_{12}^{\prime}, \bar{A}_{13}^{\prime}, \bar{A}_{12}, \bar{A}_{13}, \bar{A}_{23} \wedge \bar{A}_{23}^{\prime}, A_{1}^{\prime}, A_{1}, A_{2} \wedge A_{2}^{\prime}\right.$, $\left.A_{3} \wedge A_{3}^{\prime}\right)$. 
Example 3.2.7.a. The following $M-w f f$ is valid.

$$
\bar{M}(3,2)\left(A_{1}, A_{2}, A_{3}, \bar{A}_{12}, \bar{A}_{13}, \bar{A}_{23}\right) \rightarrow C_{1} \vee C_{2} \vee C_{3} \vee C_{4} \vee C_{5} \vee C_{6},
$$

where

(a) $\bar{A}_{12}=\bar{I} e(3,1,2)\left(\bar{A}_{12}^{\prime}, \bar{A}_{13}^{\prime}, \bar{A}_{23}^{\prime}, A_{1}^{\prime}, A_{2}^{\prime}, A_{3}^{\prime}\right)$.

(b) $C_{1}=\bar{M}(3,2)\left(A_{1} \wedge A_{1}^{\prime}, A_{2}, A_{3}, \bar{A}_{12}, \bar{A}_{13}, \bar{A}_{23}\right)$.

(c) $C_{2}=\bar{M}(3,2)\left(A_{1}, A_{2} \wedge A_{2}^{\prime}, A_{3}, \bar{A}_{12}, \bar{A}_{13}, \bar{A}_{23}\right)$.

(d) $C_{3}=\bar{M}(3,2)\left(A_{1}, A_{2}, A_{3} \wedge A_{3}^{\prime}, \bar{A}_{12}, \bar{A}_{13}, \bar{A}_{23}\right)$.

(e) $C_{4}=\bar{M}(3,2)\left(A_{1}, A_{2}, A_{3}, \bar{A}_{12} \wedge \bar{A}_{12}^{\prime}, \bar{A}_{13}, \bar{A}_{23}\right)$.

(f) $C_{5}=\bar{M}(3,2)\left(A_{1}, A_{2}, A_{3}, \bar{A}_{12}, \bar{A}_{13} \wedge \bar{A}_{13}^{\prime}, \bar{A}_{23}\right)$.

(g) $C_{6}=\bar{M}(3,2)\left(A_{1}, A_{2}, A_{3}, \bar{A}_{12}, \bar{A}_{13}, \bar{A}_{23} \wedge \bar{A}_{23}^{\prime}\right)$.

Example 3.2.7.b. The following I-wff is valid.

$$
\bar{I}(3,1,2)\left(\bar{A}_{12}, \bar{A}_{13}, \bar{A}_{23}, A_{1}, A_{2}, A_{3}\right) \rightarrow \bar{C}_{1} \vee \bar{C}_{2} \vee \bar{C}_{3} \vee \bar{C}_{4} \vee \bar{C}_{5} \vee \bar{C}_{6},
$$

where

(a) $\bar{A}_{23}=\bar{I} e(3,2,3)\left(\bar{A}_{12}^{\prime}, \bar{A}_{13}^{\prime}, \bar{A}_{23}^{\prime}, A_{1}^{\prime}, A_{2}^{\prime}, A_{3}^{\prime}\right)$.

(b) $\bar{C}_{1}=\bar{I}(3,1,2)\left(\bar{A}_{12} \wedge \bar{A}_{12}^{\prime}, \bar{A}_{13}, \bar{A}_{23}, A_{1}, A_{2}, A_{3}\right)$.

(c) $\bar{C}_{2}=\bar{I}(3,1,2)\left(\bar{A}_{12}, \bar{A}_{13} \wedge \bar{A}_{13}^{\prime}, \bar{A}_{23}, A_{1}, A_{2}, A_{3}\right)$.

(d) $\bar{C}_{3}=\bar{I}(3,1,2)\left(\bar{A}_{12}, \bar{A}_{13}, \bar{A}_{23} \wedge \bar{A}_{23}^{\prime}, A_{1}, A_{2}, A_{3}\right)$.

(e) $\bar{C}_{4}=\bar{I}(3,1,2)\left(\bar{A}_{12}, \bar{A}_{13}, \bar{A}_{23}, A_{1} \wedge A_{1}^{\prime}, A_{2}, A_{3}\right)$.

(f) $\bar{C}_{5}=\bar{I}(3,1,2)\left(\bar{A}_{12}, \bar{A}_{13}, \bar{A}_{23}, A_{1}, A_{2} \wedge A_{2}^{\prime}, A_{3}\right)$.

(g) $\quad \bar{C}_{6}=\bar{I}(3,1,2)\left(\bar{A}_{12}, \bar{A}_{13}, \bar{A}_{23}, A_{1}, A_{2}, A_{3} \wedge A_{3}^{\prime}\right)$.

\section{§3.3. DMI-Sequence and DIM-Sequence}

This section is devoted to some definitions around the notions of a DMIsequence and a DIM-sequence.

\section{Definition 3.3.1.}

(1) By a DM-set we mean a triple $(S,<, \bar{s})$, where

(a) $S$ is a set.

(b) $<$ is a strict linear order in $S$.

(c) $\bar{s} \in S$.

(2) A DM-set $\left(S^{\prime},<, \bar{s}\right)$ is called a DM-subset of a DM-set $(S,<, \bar{s})$ if $\left(S^{\prime},<\right)$ is a restriction of $(S,<)$ as an ordered set, i.e., $S^{\prime}$ is a subset of $S$ and $s^{\prime}<t^{\prime}$ in $S^{\prime}$ iff $s^{\prime}<t^{\prime}$ in $S$ for any $s^{\prime}, t^{\prime} \in S^{\prime}$.

(3) A DM-set $(S,<, \bar{s})$ is called canonical if $S=[1, n]$ for some $n$ and $<$ is 
the usual ordering of natural numbers.

(4) A DM-set $(S,<, \bar{s})$ is called finite if $S$ is finite.

\section{Definition 3.3.2.}

(1) A DM-monomorphism from a DM-set $(S,<, \bar{s})$ to a DM-set $\left(S^{\prime},<, \bar{s}^{\prime}\right)$ is an order monomorphism $f$ from $(S,<)$ to $\left(S^{\prime},<\right)$ such that $f(\bar{s})=\bar{s}^{\prime}$.

(2) A DM-isomorphism from a DM-set $(S,<, \bar{s})$ to a DM-set $\left(S^{\prime},<, \bar{s}^{\prime}\right)$ is a DI-monomorphism from $(S,<, \bar{s})$ onto $\left(S^{\prime},<, \bar{s}^{\prime}\right)$.

(3) Two DM-sets $(S,<, \bar{s})$ and $\left(S^{\prime},<, \bar{s}^{\prime}\right)$ are called isomorphic, notation: $(S,<, \bar{s}) \approx\left(S^{\prime},<, \bar{s}^{\prime}\right)$, if there exists a DM-isomorphism from one to the other.

\section{Definition 3.3.3.a.}

(1) A DMI-sequence $\alpha$ is a 5-tuple $(S,<, \bar{s}, \omega, \Omega)$, where

(a) $(S,<, \bar{s})$ is a DM-set.

(b) $\omega$ is a function from $S$ to M-WFF while $\Omega$ is a function from $I(S,<)$ to I-WFF, where M-WFF is the totality of M-wffs and I-WFF is the totality of I-wffs.

(2) A DMI-sequence $\left(S^{\prime},<, \bar{s}, \omega^{\prime}, \Omega^{\prime}\right)$ is called a DMI-subsequence of a DMI-sequence $(S,<, \bar{s}, \omega, \Omega)$ if $\left(S^{\prime},<, \bar{s}\right)$ is a DM-subset of $(S,<, \bar{s})$ and $\omega^{\prime}$ and $\Omega^{\prime}$ are the restrictions of $\omega$ and $\Omega$ to $S^{\prime}$ and $I\left(S^{\prime},<\right)$ respectively.

(3) A DMI-sequence $(S,<, \bar{s}, \omega, \Omega)$ is called finite if the cardinality of $S$ is finite.

(4) A DMI-sequence $(S,<, \bar{s}, \omega, \Omega)$ is called canonical if $(S,<, \bar{s})$ is canonical as a DM-set.

\section{Definition 3.3.3.b.}

(1) A DIM-sequence $\beta$ is a 5 -tuple $(S,<,(\bar{s}, \bar{t}), \omega, \Omega)$, where

(a) $(S,<,(\bar{s}, \bar{t}))$ is a DI-set (see Definition 2.3.1).

(b) $\omega$ is a function from $S$ to M-WFF.

(c) $\Omega$ is a function from $I(S,<)$ to I-WFF.

(2)-(4) are similar to those of Definition 3.3.3.a respectively.

\section{Definition 3.3.4.a.}

(1) A DMI-monomorphism from a DMI-sequence $(S,<, \bar{s}, \omega, \Omega)$ to a DMIsequence $\left(S^{\prime},<, \bar{s}^{\prime}, \omega^{\prime}, \Omega^{\prime}\right)$ is a DM-monomorphism $f$ from $(S,<, \bar{s})$ to $\left(S^{\prime},<, \bar{s}^{\prime}\right)$ such that $\omega=\omega^{\prime} \circ f$ and $\Omega=\Omega^{\prime} \circ f$, i.e., $\omega(s)=\omega^{\prime}(f(s))$ for any $s \in S$ and $\Omega(s, t)=\Omega^{\prime}(f(s), f(t))$ for any $(s, t) \in I(S,<)$. 
(2) A DMI-monomorphism $f$ is called a DMI-isomorphism if $f$ is onto.

(3) Two DMI-sequences $(S,<, \bar{s}, \omega, \Omega)$ and $\left(S^{\prime},<, \bar{s}^{\prime}, \omega^{\prime}, \Omega^{\prime}\right)$ are called isomorphic, notation: $(S,<, \bar{s}, \omega, \Omega) \approx\left(S^{\prime},<, \bar{s}^{\prime}, \omega^{\prime}, \Omega^{\prime}\right)$, if there exists a DMI-isomorphism from one to the other.

Definition 3.3.4.b.

(1) A DIM-monomorphism from a DIM-sequence $(S,<,(\bar{s}, \bar{t}), \omega, \Omega)$ to a DIM-sequence $\left(S^{\prime},<,\left(\bar{s}^{\prime}, \bar{t}^{\prime}\right), \omega^{\prime}, \Omega^{\prime}\right)$ is a DI-monomorphism $(S,<,(\bar{s}, \bar{t}))$ to $\left(S^{\prime},<,\left(\bar{s}^{\prime}, \bar{t}^{\prime}\right)\right)$ such that $\omega=\omega^{\prime} \circ f$ and $\Omega=\Omega^{\prime} \circ f$, i.e., $\omega(s)=\omega^{\prime}(f(s))$ for any $s \in S$ and $\Omega(s, t)=\Omega^{\prime}(f(s), f(t))$ for any $(s, t) \in I(S,<)$.

(2) and (3) are similar to those of Definition 3.3.4.a, respectively.

Definition 3.3.5.a. Let $\alpha=(S,<, \bar{s}, \omega, \Omega)$ and $\alpha^{\prime}=\left(S^{\prime},<, \bar{s}^{\prime}, \omega^{\prime}, \Omega^{\prime}\right)$ be DMI-sequences and $s_{1} \in S$. Then a sum of $\alpha$ and $\alpha^{\prime}$ with respect to $s_{1}$ is a triple $\left(\alpha^{\prime \prime}, f, g\right)$ (or simply, $\left.\alpha^{\prime \prime}\right)$, where

(a) $\alpha^{\prime \prime}$ is a DMI-sequence $\left(S^{\prime \prime},<, \bar{s}^{\prime \prime}, \omega^{\prime \prime}, \Omega^{\prime \prime}\right)$.

(b) $f$ is a DM-monomorphism from $(S,<, \bar{s})$ to $\left(S^{\prime \prime},<, \bar{s}^{\prime \prime}\right)$.

(c) $g$ is an order monomorphism from $\left(S^{\prime},<\right)$ to $\left(S^{\prime \prime},<\right)$ such that $g\left(\bar{s}^{\prime}\right)$ $=f\left(s_{1}\right)$.

(d) $S^{\prime \prime}=f(S) \cup g\left(S^{\prime}\right)$.

(e) $\omega^{\prime \prime}$ is defined as follows:

(i) Suppose $s^{\prime \prime} \in S^{\prime \prime}$ is such that $s^{\prime \prime}=f(s)=g\left(s^{\prime}\right)$ for some $s \in S$ and $s^{\prime} \in S^{\prime}$. Then $\omega^{\prime \prime}\left(s^{\prime \prime}\right)=\omega(s) \wedge \omega^{\prime}\left(s^{\prime}\right)$, i.e., $\omega^{\prime \prime}\left(s^{\prime \prime}\right)$ is the conjunction of $\omega(s)$ and $\omega^{\prime}\left(s^{\prime}\right)$.

(ii) Suppose $s^{\prime \prime} \in S^{\prime \prime}$ is such that $s^{\prime \prime}=f(s)$ for some $s \in S$ and $s^{\prime \prime} \neq g\left(s^{\prime}\right)$ for any $s^{\prime} \in S^{\prime}$. Then $\omega^{\prime \prime}\left(s^{\prime \prime}\right)=\omega(s)$.

(iii) Suppose $s^{\prime \prime} \in S^{\prime \prime}$ is such that $s^{\prime \prime} \neq f(s)$ for any $s \in S$ and $s^{\prime \prime}=g\left(s^{\prime}\right)$ for some $s^{\prime} \in S^{\prime}$. Then $\omega^{\prime \prime}\left(s^{\prime \prime}\right)=\omega^{\prime}\left(s^{\prime}\right)$.

(iv) Suppose $s^{\prime \prime} \in S^{\prime \prime}$ is such that $s^{\prime \prime} \neq f(s)$ for any $s \in S$ and $s^{\prime \prime} \neq g\left(s^{\prime}\right)$ for any $s^{\prime} \in S^{\prime}$. Then $\omega^{\prime \prime}\left(s^{\prime \prime}\right)=T$.

(f) $\Omega^{\prime \prime}$ is defined as follows:

(i) Suppose $\left(s^{\prime \prime}, t^{\prime \prime}\right) \in I\left(S^{\prime \prime},<\right)$ is such that $\left(s^{\prime \prime}, t^{\prime \prime}\right)=(f(s), f(t))$ $=\left(g\left(s^{\prime}\right), g\left(t^{\prime}\right)\right)$ for some $(s, t) \in I(S,<)$ and $\left(s^{\prime}, t^{\prime}\right) \in I\left(S^{\prime},<\right)$. Then $\Omega^{\prime \prime}\left(s^{\prime \prime}, t^{\prime \prime}\right)=\Omega(s, t) \wedge \Omega^{\prime}\left(s^{\prime}, t^{\prime}\right)$.

(ii) Suppose $\left(s^{\prime \prime}, t^{\prime \prime}\right) \in I\left(S^{\prime \prime},<\right)$ is such that $\left(s^{\prime \prime}, t^{\prime \prime}\right)=(f(s), f(t))$ for some $(s, t) \in I(S,<)$ and $\left(S^{\prime \prime}, t^{\prime \prime}\right) \neq\left(g\left(s^{\prime}\right), g\left(t^{\prime}\right)\right)$ for any $\left(s^{\prime}, t^{\prime}\right)$ $\in I\left(S^{\prime},<\right)$. Then $\Omega^{\prime \prime}\left(s^{\prime \prime}, t^{\prime \prime}\right)=\Omega(s, t)$. 
(iii) Suppose $\left(s^{\prime \prime}, t^{\prime \prime}\right) \in I\left(S^{\prime \prime},<\right)$ is such that $\left(s^{\prime \prime}, t^{\prime \prime}\right) \neq(f(s), f(t))$ for any $(s, t) \in I(S,<)$ and $\left(s^{\prime \prime}, t^{\prime \prime}\right)=\left(g\left(s^{\prime}\right), g\left(t^{\prime}\right)\right)$ for some $\left(s^{\prime}, t^{\prime}\right)$ $\in I\left(S^{\prime},<\right)$. Then $\Omega^{\prime \prime}\left(s^{\prime \prime}, t^{\prime \prime}\right)=\Omega^{\prime}\left(s^{\prime}, t^{\prime}\right)$.

(iv) Suppose $\left(s^{\prime \prime}, t^{\prime \prime}\right) \in I\left(S^{\prime \prime},<\right)$ is such that $\left(s^{\prime \prime}, t^{\prime \prime}\right) \neq(f(s), f(t))$ for any $(s, t) \in I(S,<)$ and $\left(s^{\prime \prime}, t^{\prime \prime}\right) \neq\left(g\left(s^{\prime}\right), g\left(t^{\prime}\right)\right)$ for any $\left(s^{\prime}, t^{\prime}\right)$ $\in I\left(S^{\prime},<\right)$. Then $\Omega^{\prime \prime}\left(s^{\prime \prime}, t^{\prime \prime}\right)=\bar{T}$.

We denote by $C S\left(\alpha, \alpha^{\prime}, s_{1}\right)$ the totality of canonical DMI-sequences that are sums of $\alpha$ and $\alpha^{\prime}$ with respect to $s_{1}$, provided $\alpha$ and $\alpha^{\prime}$ are finite.

Definition 3.3.5.b. Suppose that:

(a) $\alpha=(S,<, \bar{s}, \omega, \Omega)$ is a DMI-sequence.

(b) $\beta^{\prime}=\left(S,<,\left(\bar{s}^{\prime}, \bar{t}^{\prime}\right), \omega^{\prime}, \Omega^{\prime}\right)$ is a DIM-sequence.

(c) $\left(s_{1}, t_{1}\right) \in I(S,<)$.

Then a sum of $\alpha$ and $\beta^{\prime}$ with respect to $\left(s_{1}, t_{1}\right)$ is a triple $\left(\alpha^{\prime \prime}, f, g\right)$ (or simply, $\left.\alpha^{\prime \prime}\right)$, where

(a) $\alpha^{\prime \prime}$ is a DMI-sequence $\left(S^{\prime \prime},<, \bar{s}^{\prime \prime}, \omega^{\prime \prime}, \Omega^{\prime \prime}\right)$.

(b) $f$ is a DM-monomorphism from $(S,<, \bar{s})$ to $\left(S^{\prime \prime},<, \bar{s}^{\prime \prime}\right)$.

(c) $g$ is an order monomorphism from $\left(S^{\prime},<\right)$ to $\left(S^{\prime \prime},<\right)$ such that $g\left(\bar{s}^{\prime}\right)$ $=f\left(s_{1}\right)$ and $g\left(\bar{t}^{\prime}\right)=f\left(t_{1}\right)$.

(d)-(f) are similar to those of Definition 3.3.5.a.

We denote by $C S\left(\alpha, \beta^{\prime}, s_{1}, t_{1}\right)$ the totality of canonical DMI-sequences that are sums of $\alpha$ and $\beta^{\prime}$ with respect to $\left(s_{1}, t_{1}\right)$, provided $\alpha$ and $\beta^{\prime}$ are finite.

Definition 3.3.5.c. Suppose that:

(a) $\beta=(S,<,(\bar{s}, \bar{t}), \omega, \Omega)$ is a DIM-sequence.

(b) $\alpha^{\prime}=\left(S^{\prime},<, \bar{s}^{\prime}, \omega^{\prime}, \Omega^{\prime}\right)$ is a DMI-sequence.

(c) $s_{1} \in S$.

Then a sum of $\beta$ and $\alpha^{\prime}$ with respect to $s$ is a triple $\left(\beta^{\prime \prime}, f, g\right)$ (or simply, $\left.\beta^{\prime \prime}\right)$, where

(a) $\beta^{\prime \prime}$ is a DIM-sequence $\left(S^{\prime \prime},<,\left(\bar{s}^{\prime \prime}, \bar{t}^{\prime \prime}\right), \omega^{\prime \prime}, \Omega^{\prime \prime}\right)$.

(b) $f$ is a DI-monomorphism from $(S,<,(\bar{s}, \bar{t}))$ to $\left(S^{\prime \prime},<,\left(\bar{s}^{\prime \prime}, \bar{t}^{\prime \prime}\right)\right)$.

(c) $g$ is an order monomorphism from $\left(S^{\prime},<\right)$ to $\left(S^{\prime \prime},<\right)$ such that $g\left(\bar{s}^{\prime}\right)$ $=f\left(s_{1}\right)$.

(d)-(f) are similar to those of Definition 3.3.5.a.

We denote by $C S\left(\beta, \alpha^{\prime}, s_{1}\right)$ the totality of canonical DIM-sequences that are 
sums of $\beta$ and $\alpha^{\prime}$ with respect to $s_{1}$, provided $\beta$ and $\alpha^{\prime}$ are finite.

Definition 3.3.5.d. Let $\beta=(S,<,(\bar{s}, \bar{t}), \omega, \Omega)$ and $\beta^{\prime}=\left(S^{\prime},<,\left(\bar{s}^{\prime}, \bar{t}^{\prime}\right), \omega^{\prime}\right.$, $\left.\Omega^{\prime}\right)$ be DIM-sequences and $\left(s_{1}, t_{1}\right) \in I(S,<)$. Then a sum of $\beta$ and $\beta^{\prime}$ with respect to $\left(s_{1}, t_{1}\right)$ is a triple $\left(\beta^{\prime \prime}, f, g\right)$ (or simply, $\left.\beta^{\prime \prime}\right)$, where

(a) $\beta^{\prime \prime}$ is a DIM-sequence $\left(S^{\prime \prime},<,\left(\bar{s}^{\prime \prime}, \bar{t}^{\prime \prime}\right), \omega^{\prime \prime}, \Omega^{\prime \prime}\right)$.

(b) $f$ is a DI-monomorphism from $(S,<,(\bar{s}, \bar{t}))$ to $\left(S^{\prime \prime},<,\left(\bar{s}^{\prime \prime}, \bar{t}^{\prime \prime}\right)\right)$.

(c) $g$ is an order monomorphism from $\left(S^{\prime},<\right)$ to $\left(S^{\prime \prime},<\right)$ such that $g\left(\bar{s}^{\prime}\right)=f\left(s_{1}\right)$ and $g\left(\bar{t}^{\prime}\right)=f\left(t_{1}\right)$.

(d)-(f) are similar to those of Definition 3.3.5.a.

We denote by $\operatorname{CS}\left(\beta, \beta^{\prime}, s_{1}, t_{1}\right)$ the totality of canonical DIM-sequences that are sums of $\beta$ and $\beta^{\prime}$ with respect to $\left(s_{1}, t_{1}\right)$, provided $\beta$ and $\beta^{\prime}$ are finite.

Definition 3.3.6.a. Let $\alpha=(S,<, \bar{s}, \omega, \Omega)$ and $\alpha^{\prime}=\left(S^{\prime},<, \bar{s}^{\prime}, \omega^{\prime}, \Omega^{\prime}\right)$ be DMI-sequences and $f$ be an order monomorphism from $\left(S^{\prime},<\right)$ to $(S,<)$. Then the immersion of $\alpha^{\prime}$ into $\alpha$ at $s_{1}^{\prime} \in S^{\prime}$ with respect to $f$ is the DMI-sequence $\alpha^{\prime \prime}=\left(S,<, \bar{s}, \omega^{\prime \prime}, \Omega\right)$, where $\omega^{\prime \prime}\left(f\left(s_{1}^{\prime}\right)\right)=\omega\left(f\left(s_{1}^{\prime}\right)\right) \wedge \omega^{\prime}\left(s_{1}^{\prime}\right)$ while $\omega^{\prime \prime}(s)=\omega(s)$ for any other $s \in S$. The immersion of $\alpha^{\prime}$ into $\alpha$ at $\left(s_{1}^{\prime}, t_{1}^{\prime}\right) \in I\left(S^{\prime},<\right)$ with respect to $f$ is the DMI-sequence $\alpha^{\prime \prime}=\left(S,<, \bar{s}, \omega, \Omega^{\prime \prime}\right)$, where $\Omega^{\prime \prime}\left(f\left(s_{1}^{\prime}\right), f\left(t_{1}^{\prime}\right)\right)=\Omega\left(f\left(s_{1}^{\prime}\right)\right.$, $\left.f\left(t_{1}^{\prime}\right)\right) \wedge \Omega\left(s_{1}^{\prime}, t_{1}^{\prime}\right)$ while $\Omega^{\prime \prime}(s, t)=\Omega(s, t)$ for any other $(s, t) \in I(S,<)$. We denote by $\operatorname{IM}\left(\alpha, \alpha^{\prime}, f\right)$ the totality of immersions of $\alpha$ into $\alpha^{\prime}$ with respect to $f$.

Definition 3.3.6.b-Definition 3.3.6.d are such as are expected.

\section{§3.4. Formal System IM}

The main purpose of this section is to present the formal system IM. To do it smoothly, we need some notational conventions.

Given a finite DMI-sequence $\alpha=(S,<, \bar{s}, \omega, \Omega)$, we can easily find out the canonical DMI-sequence $\alpha^{\prime}=\left([1, n],<, z, \omega^{\prime}, \Omega^{\prime}\right)$ such that $\alpha^{\prime} \approx \alpha$. We will denote the M-wff $\bar{M}(n, z)\left(\left\{\omega^{\prime}(i)\right\},\left\{\Omega^{\prime}(i, j)\right\}\right)$ by $\bar{M}(n, z)(\alpha)$ or $\bar{M}(),(\alpha)$. We will denote the M-wff $\bar{M} e(n, z)\left(\left\{\omega^{\prime}(i)\right\},\left\{\Omega^{\prime}(i, j)\right\}\right)$ by $\bar{M} e(n, z)(\alpha)$ or $\bar{M} e(),(\alpha)$. Given a finite DIM-sequence $\beta=(S,<,(\bar{s}, \bar{t}), \omega, \Omega)$, the I-wffs $\bar{I}(n, x, y)(\beta)$ $=\bar{I}(,),(\beta)$ and $\bar{I} e(n, x, y)(\beta)=\bar{I} e(,),(\beta)$ are defined similarly.

Definition 3.4.1. Our formal system IM consists of the following rules and axioms: 
Rules:

$$
\begin{aligned}
& \text { (MPM) } \frac{\vdash A \vdash A \rightarrow B}{\vdash B} \\
& \text { (MPI) } \quad \frac{\vdash \bar{A} \vdash \bar{A} \rightarrow \bar{B}}{\vdash \bar{B}} \\
& \text { (RMD) } \frac{\vdash A_{i} \leftrightarrow B_{i} \quad(1 \leq i \leq n) \quad \vdash \bar{A}_{i j} \leftrightarrow \bar{B}_{i j} \quad(1 \leq i<j \leq n)}{\vdash \bar{M}(n, z)\left(\left\{A_{i}\right\},\left\{\bar{A}_{i j}\right\} \leftrightarrow \bar{M}(n, z)\left(\left\{B_{i}\right\},\left\{\bar{B}_{i j}\right\}\right)\right.} \\
& \text { (RID) } \frac{\vdash \bar{A}_{i j} \leftrightarrow \bar{B}_{i j}(1 \leq i<j \leq n) \quad \vdash A_{i} \leftrightarrow B_{i}(1 \leq i \leq n)}{\vdash \bar{I}(n, x, y)\left(\left\{\bar{A}_{i j}\right\},\left\{A_{i}\right\}\right) \leftrightarrow \bar{I}(n, x, y)\left(\left\{\bar{B}_{i j}\right\},\left\{B_{i}\right\}\right)} \\
& (\mathrm{RMMN}) \frac{\vdash A}{\vdash \bar{M} e(n, z)\left(\left\{A_{i}\right\},\left\{\bar{A}_{i j}\right\}\right)}, \\
& \text { where } A=A_{i} \text { for some } i \text {. } \\
& \text { (RMIN) } \frac{\vdash \bar{A}}{\vdash \bar{M} e(n, z)\left(\left\{A_{i}\right\},\left\{\bar{A}_{i j}\right\}\right)}, \\
& \text { where } A=A_{i j} \text { for some }(i, j) \text {. } \\
& (\mathrm{RIMN}) \frac{\vdash A}{\vdash \bar{I} e(n, x, y)\left(\left\{\bar{A}_{i j}\right\},\left\{A_{i}\right\}\right)}, \\
& \text { where } A=A_{i} \text { for some } i \text {. } \\
& \text { (RIIN) } \frac{\vdash \bar{A}}{\vdash \bar{I} e(n, x, y)\left(\left\{\bar{A}_{i j}\right\},\left\{A_{i}\right\}\right)} \text {, } \\
& \text { where } \bar{A}=\bar{A}_{i j} \text { for some }(i, j) \text {. }
\end{aligned}
$$

Axioms:

$$
\begin{array}{ll}
\text { (AM1) } & A \rightarrow(B \rightarrow A) \\
\text { (AI1) } & \bar{A} \rightarrow(\bar{B} \rightarrow \bar{A}) \\
\text { (AM2) } & (A \rightarrow(B \rightarrow C)) \rightarrow((A \rightarrow B) \rightarrow(A \rightarrow C)) \\
\text { (AI2) } & (\bar{A} \rightarrow(\bar{B} \rightarrow \bar{C})) \rightarrow((\bar{A} \rightarrow \bar{B}) \rightarrow(\bar{A} \rightarrow \bar{C})) \\
\text { (AM3) } & (\neg B \rightarrow \neg A) \rightarrow(A \rightarrow B) \\
\text { (AI3) } & (\neg \bar{B} \rightarrow \neg \bar{A}) \rightarrow(\bar{A} \rightarrow \bar{B}) \\
\text { (M0) } & \bar{M}(1,1)(T) \\
\text { (I0) } & \bar{I}(2,1,2)(\bar{T}, T, T) \\
\text { (MM1) } & \bar{M} e(n, z)\left(\left\{A_{i}\right\},\left\{\bar{A}_{i j}\right\}\right) \wedge \bar{M} e(n, z)\left(\left\{A_{i}^{\prime}\right\},\left\{\bar{A}_{i j}^{\prime}\right\}\right) \rightarrow \\
& \bar{M} e(n, z)\left(\left\{A_{i}^{\prime \prime}\right\},\left\{\bar{A}_{i j}^{\prime \prime}\right\}\right), \text { where }
\end{array}
$$

(a) for some $i_{1}, A_{i_{1}}=A_{i_{1}}^{\prime} \rightarrow A_{i_{1}}^{\prime \prime}$.

(b) for any other $i, A_{i}=A_{i}^{\prime}=A_{i}^{\prime \prime}$. 
(c) for any $(i, j), \bar{A}_{i j}=\bar{A}_{i j}^{\prime}=\bar{A}_{i j}^{\prime \prime}$.

(MI1) $\bar{M} e(n, z)\left(\left\{A_{i}\right\},\left\{\bar{A}_{i j}\right\}\right) \wedge \bar{M} e(n, z)\left(\left\{A_{i}^{\prime}\right\},\left\{\bar{A}_{i j}^{\prime}\right\}\right) \rightarrow$ $\bar{M} e(n, z)\left(\left\{A_{i}^{\prime \prime}\right\},\left\{\bar{A}_{i j}^{\prime \prime}\right\}\right)$, where

(a) for any $i, A_{i}=A_{i}^{\prime}=A_{i}^{\prime \prime}$.

(b) for some $\left(i_{1}, j_{1}\right)$, $\bar{A}_{i_{1} j_{1}}=\bar{A}_{i_{1} j_{1}}^{\prime} \rightarrow \bar{A}_{i_{1} j_{1}}^{\prime \prime}$.

(c) for any other $(i, j), \bar{A}_{i j}=\bar{A}_{i j}^{\prime}=\bar{A}_{i j}^{\prime \prime}$.

(IM1) $\bar{I} e(n, x, y)\left(\left\{\bar{A}_{i j}\right\},\left\{A_{j}\right\}\right) \wedge \bar{I} e(n, x, y)\left(\left\{\bar{A}_{i j}^{\prime}\right\},\left\{A_{i}^{\prime}\right\}\right) \rightarrow$ $\bar{I} e(n, x, y)\left(\left\{\bar{A}_{i j}^{\prime \prime}\right\},\left\{A_{i}^{\prime \prime}\right\}\right)$, where

(a) for any $(i, j), \bar{A}_{i j}=\bar{A}_{i j}^{\prime}=\bar{A}_{i j}^{\prime \prime}$.

(b) for some $i_{1}, A_{i_{1}}=A_{i_{1}}^{\prime} \rightarrow A_{i_{1}}^{\prime \prime}$.

(c) for any other $i, A_{i}=A_{i}^{\prime}=A_{i}^{\prime \prime}$.

(II1) $\bar{I} e(n, x, y)\left\{\left(\bar{A}_{i j}\right\},\left\{A_{i}\right\}\right) \wedge \bar{I} e(n, x, y)\left(\left\{\bar{A}_{i j}^{\prime}\right\},\left\{A_{i}^{\prime}\right\}\right) \rightarrow$ $\bar{I} e(n, x, y)\left(\left\{\bar{A}_{i j}^{\prime \prime}\right\},\left\{A_{i}^{\prime \prime}\right\}\right)$, where
(a) for some $\left(i_{1}, j_{1}\right)$, $\bar{A}_{i_{1} j_{1}}=\bar{A}_{i_{1} j_{1}}^{\prime} \rightarrow \bar{A}_{i_{1} j_{1}}^{\prime \prime}$ 。
(b) for any other $(i, j), \bar{A}_{i j}=\bar{A}_{i j}^{\prime}=\bar{A}_{i j}^{\prime \prime}$.
(c) for any $i, A_{i}=A_{i}^{\prime}=A_{i}^{\prime \prime}$.

(M2) $\bar{M}(n, z)\left(\left\{A_{i}\right\},\left\{\bar{A}_{i j}\right\}\right) \leftrightarrow B \wedge \bar{M}(n, z)\left(\left\{A_{i}^{\prime}\right\},\left\{\bar{A}_{i j}^{\prime}\right\}\right)$, where

(a) $A_{z}=B \wedge A_{z}^{\prime}$.

(b) for any other $i, A_{i}=A_{i}^{\prime}$.

(c) for any $(i, j), \bar{A}_{i j}=\bar{A}_{i j}^{\prime}$.

(I2) $\quad \vec{I}(n, x, y)\left(\left\{\bar{A}_{i j}\right\},\left\{A_{i}\right\}\right) \leftrightarrow \bar{B} \wedge \bar{I}(n, x, y)\left(\left\{\bar{A}_{i j}^{\prime}\right\},\left\{A_{i}^{\prime}\right\}\right)$, where

(a) $\bar{A}_{x y}=\bar{B} \wedge \bar{A}_{x y}^{\prime}$ 。

(b) for any other $(i, j), \bar{A}_{i j}=\bar{A}_{i j}^{\prime}=\bar{A}_{i j}^{\prime \prime}$ 。

(c) for any $i, A_{i}=A_{i}^{\prime}=A_{i}^{\prime \prime}$.

(M3) $\bar{M}(n, z)(\alpha) \rightarrow \bar{M}\left(n^{\prime}, z^{\prime}\right)\left(\alpha^{\prime}\right)$, where

(a) $\alpha$ is a finite DMI-sequence.

(b) $\alpha^{\prime}$ is a DMI-subsequence of $\alpha$.

(I3) $\quad \bar{I}(n, x, y)(\beta) \rightarrow \bar{I}\left(n^{\prime}, x^{\prime}, y^{\prime}\right)\left(\beta^{\prime}\right)$, where 
(a) $\beta$ is a finite DIM-sequence.

(b) $\beta^{\prime}$ is a DIM-subsequence of $\beta$.

(MM4) $\bar{M}(n, z)\left(\left\{A_{i}\right\},\left\{\bar{A}_{i j}\right\}\right) \rightarrow \vee_{\gamma \in C S\left(\alpha, \alpha^{\prime}, i_{1}\right)} \bar{M}(),(\gamma)$,

where

(a) for some $i_{1}, A_{i_{1}}$ is of the form

$M\left(n^{\prime}, z^{\prime}\right)\left(\left\{A_{i}^{\prime}\right\},\left\{\bar{A}_{i j}^{\prime}\right\}\right)$.

(b) $\alpha=([1, n],<, z, \omega, \Omega)$, where $\omega(i)=A_{i}$ for any $i$ and $\Omega(i, j)$ $=\bar{A}_{i j}$ for any $(i, j)$.

(c) $\alpha^{\prime}=\left(\left[1, n^{\prime}\right],<, z^{\prime}, \omega^{\prime}, \Omega^{\prime}\right)$, where $\omega^{\prime}(i)=A_{i}^{\prime}$ for any $i$ and $\Omega^{\prime}(i, j)=\bar{A}_{i j}^{\prime}$ for any $(i, j)$.

(MI4) $\bar{M}(n, z)\left(\left\{A_{i}\right\},\left\{\bar{A}_{i j}\right\}\right) \rightarrow \vee_{\gamma \in C S\left(\alpha, \beta^{\prime}, i_{1}, j_{1}\right)} \bar{M}(),(\gamma)$,

where

(a) for some $\left(i_{1}, j_{1}\right), \bar{A}_{i_{1} j_{1}}$ is of the form $I\left(n^{\prime}, 1^{\prime}, r^{\prime}\right)\left(\left\{\bar{A}_{i j}^{\prime}\right\},\left\{A_{i}^{\prime}\right\}\right)$.

(b) $\alpha=([1, n],<, z, \omega, \Omega)$, where $\omega(i)=A_{i}$ for any $i$ and $\Omega(i, j)=\bar{A}_{i j}$ for any $(i, j)$.

(c) $\beta^{\prime}=\left(\left[1, n^{\prime}\right],<,\left(1^{\prime}, r^{\prime}\right), \omega^{\prime}, \Omega^{\prime}\right)$, where $\omega^{\prime}(i)=A_{i}^{\prime}$ for any $i$ and $\Omega^{\prime}(i, j)=\bar{A}_{i j}^{\prime}$ for any $(i, j)$.

(IM4) $\bar{I}(n, x, y)\left(\left\{\bar{A}_{i j}\right\},\left\{A_{i}\right\}\right) \rightarrow \vee_{\gamma \in C S\left(\beta, \alpha^{\prime}, i_{1}\right)} \bar{I}(,),(\gamma)$, where

(a) for some $i_{1}, A_{i_{1}}$ is of the form $M\left(n^{\prime}, z^{\prime}\right)\left(\left\{A_{i}^{\prime}\right\},\left\{\bar{A}_{i j}^{\prime}\right\}\right)$.

(b) $\beta=([1, n],<,(1, r), \omega, \Omega)$, where $\omega(i)=A_{i}$ for any $i$ and $\Omega(i, j)=\bar{A}_{i j}$ for any $(i, j)$.

(c) $\alpha^{\prime}=\left(\left[1, n^{\prime}\right],<, z^{\prime}, \omega^{\prime}, \Omega^{\prime}\right)$, where $\omega^{\prime}(i)=A_{i}^{\prime}$ for any $i$ and $\Omega^{\prime}(i, j)=\bar{A}_{i j}^{\prime}$ for any $(i, j)$.

(II4) $\quad \bar{I}(n, x, y)\left(\left\{\bar{A}_{i j}\right\},\left\{A_{i}\right\}\right) \rightarrow \vee_{\gamma \in C S\left(\beta, \beta^{\prime}, i_{1}, j_{1}\right)} \bar{I}(,),(\gamma)$, where

(a) for some $\left(i_{1}, j_{1}\right), \bar{A}_{i_{1} j_{1}}$ is of the form $I\left(n^{\prime}, x^{\prime}, y^{\prime}\right)\left(\left\{\bar{A}_{i j}^{\prime}\right\},\left\{A_{i}^{\prime}\right\}\right)$.

(b) $\beta=([1, n],<,(x, y), \omega, \Omega)$, where $\omega(i)=A_{i}$ for any $i$ and $\Omega(i, j)=\bar{A}_{i j}$ for any $(i, j)$.

(c) $\beta^{\prime}=\left(\left[1, n^{\prime}\right],<,\left(1^{\prime}, r^{\prime}\right), \omega^{\prime}, \Omega^{\prime}\right)$, where $\omega^{\prime}(i)=A_{i}$ for any $i$ and $\Omega^{\prime}(i, j)=\bar{A}_{i, j}^{\prime}$ for any $(i, j)$. 
(MM5) $\bar{M}(n, z)\left(\left\{A_{i}\right\},\left\{\bar{A}_{i j}\right\}\right) \rightarrow \vee_{\gamma \in I M\left(\alpha, \alpha^{\prime}, f\right)} \bar{M}(),(\gamma)$, where
(a) for some $i_{1}, A_{i_{1}}$ is of the form
$B \wedge \bar{M} e\left(n^{\prime}, z^{\prime}\right)\left(\left\{A_{i}^{\prime}\right\},\left\{\bar{A}_{i j}^{\prime}\right\}\right)$.
(b) $\alpha=([1, n],<, z, \omega, \Omega)$, where $\omega(i)=A_{i}$ for any $i$ and $\Omega(i, j)$
$=\bar{A}_{i j}$ for any $(i, j)$.
(c) $\alpha^{\prime}=\left(\left[1, n^{\prime}\right],<, z^{\prime}, \omega^{\prime}, \Omega^{\prime}\right)$, where $\omega^{\prime}(i)=A_{i}^{\prime}$ for any $i$ and $\Omega^{\prime}(i, j)=\bar{A}_{i j}^{\prime}$ for any $(i, j)$.
(d) $f$ is an order monomorphism from $\left(\left[1, n^{\prime}\right],<\right)$ to $([1, n],<)$ such that $f\left(z^{\prime}\right)=i_{1}$.

(MI5) $\bar{M}(n, z)\left(\left\{A_{i}\right\},\left\{\bar{A}_{i j}\right\}\right) \rightarrow \vee_{\gamma \in I M\left(\alpha, \beta^{\prime}, f\right)} \bar{M}(),(\gamma)$,

where
(a) for some $\left(i_{1}, j_{1}\right), \bar{A}_{i_{1} j_{1}}$ is of the form $\bar{B} \wedge \bar{I} e\left(n^{\prime}, x^{\prime}, y^{\prime}\right)\left(\left\{\bar{A}_{i j}^{\prime}\right\},\left\{A_{i}^{\prime}\right\}\right)$.
(b) $\alpha=([1, n],<, z, \omega, \Omega)$, where $\omega(i)=A_{i}$ for any $i$ and $\Omega(i, j)$ $=\bar{A}_{i j}$ for any $(i, j)$.
(c) $\beta^{\prime}=\left(\left[1, n^{\prime}\right],<,\left(x^{\prime}, y^{\prime}\right), \omega^{\prime}, \Omega^{\prime}\right)$, where $\omega^{\prime}(i)=A_{i}^{\prime}$ for any $i$ and $\Omega^{\prime}(i, j)=\bar{A}_{i j}^{\prime}$ for any $(i, j)$.
(d) $f$ is an order monomorphism from $\left(\left[1, n^{\prime}\right],<\right)$ to $([1, n],<)$ such that $f\left(x^{\prime}\right)=i_{1}$ and $f\left(y^{\prime}\right)=j_{1}$.

(IM5) $\quad \bar{I}(n, x, y)\left(\left\{\bar{A}_{i j}\right\},\left\{A_{i}\right\}\right) \rightarrow \vee_{\gamma \in I M\left(\beta, \alpha^{\prime}, f\right)} \bar{I}(,),(\gamma)$,

where
(a) for some $i_{1}, A_{i_{1}}$ is of the form $B \wedge \bar{M} e\left(n^{\prime}, z^{\prime}\right)\left(\left\{A_{i}^{\prime}\right\},\left\{\bar{A}_{i j}^{\prime}\right\}\right)$.
(b) $\beta=([1, n],<,(x, y), \omega, \Omega)$, where $\omega(i)=A_{i}$ for any $i$ and $\Omega(i, j)=\bar{A}_{i j}$ for any $(i, j)$.
(c) $\alpha^{\prime}=\left(\left[1, n^{\prime}\right],<, z^{\prime}, \omega^{\prime}, \Omega^{\prime}\right)$, where $\omega^{\prime}(i)=A_{i}^{\prime}$ for any $i$ and $\Omega^{\prime}(i, j)=\bar{A}_{i j}^{\prime}$ for any $(i, j)$.
(d) $f$ is an order monomorphism from $\left(\left[1, n^{\prime}\right],<\right)$ to $([1, n],<)$ such that $f\left(z^{\prime}\right)=i_{1}$.

(II5) $\quad \bar{I}(n, x, y)\left(\left\{\bar{A}_{i j}\right\},\left\{A_{i}\right\}\right) \rightarrow \vee_{\gamma \in I M\left(\beta, \beta^{\prime}, f\right)} \bar{I}(,),(\gamma)$, where
(a) for some $\left(i_{1}, j_{1}\right), \bar{A}_{i_{1} j_{1}}$ is of the form $\bar{B} \wedge \bar{I} e\left(n^{\prime}, x^{\prime}, y^{\prime}\right)\left(\left\{\bar{A}_{i j}^{\prime}\right\},\left\{A_{i}^{\prime}\right\}\right)$. 
(b) $\beta=([1, n],<,(x, y), \omega, \Omega)$, where $\omega(i)=A_{i}$ for any $i$ and $\Omega(i, j)=\bar{A}_{i j}$ for any $(i, j)$.

(c) $\beta^{\prime}=\left(\left[1, n^{\prime}\right],<,\left(x^{\prime}, y^{\prime}\right), \omega^{\prime}, \Omega^{\prime}\right)$, where $\omega^{\prime}(i)=A_{i}^{\prime}$ for any $i$ and $\Omega^{\prime}(i, j)=\bar{A}_{i j}^{\prime}$ for any $(i, j)$.

(d) $f$ is an order monomorphism from $\left(\left[1, n^{\prime}\right],<\right)$ to $([1, n],<)$ such that $f\left(x^{\prime}\right)=i_{1}$ and $f\left(y^{\prime}\right)=j_{1}$.

Since the above list of rules and axioms of IM is parallel to that of I given in Definition 2.4.1, any explanation on the above list may presumably be redundant.

\section{§3.5. The Soundness and Completeness of IM}

Theorem 3.5.1 (Soundness of IM).

(1) For any M-wff $A, \vDash A$ provided $\vdash A$.

(2) For any I-wff $\bar{A}, \vDash \bar{A}$ provided $\vdash \bar{A}$.

Proof. By induction on the construction of the proof.

Theorem 3.5.2 (Completeness of IM).

(1) An M-theory $\Sigma$ is consistent iff it is realizable.

(2) An I-theory $\bar{\Sigma}$ is consistent iff it is realizable.

\section{Corollary 3.5.3.}

(1) For any M-wff $A, \vdash A$ iff $\vDash A$.

(2) For any I-wff $\bar{A}, \vdash \bar{A}$ iff $\vDash \bar{A}$.

Since the outline of the proof of Theorem 3.5.2 is parallel to that of Theorem 2.5.2 and is more complicated mainly in notation or terminology, it is left to the reader as an exercise.

\section{Chapter 4}

\section{Some Applications}

\section{§4.1. Some Interesting Tense Operators}

\section{Definable within IM}

The main objective of this section is to show that various interesting tense operators discussed in the literature are definable within our formal system IM. First of all, we show that tense operators corresponding to Bruce's time-segment 
relations are definable within IM.

Definition 4.1.1 (BEFORE).

(1) (a) $(I ; M)-B E F O R E(\bar{A})$ is an abbreviation of $\bar{M}(3,1)(T, T, T, \bar{T}, \bar{T}, \bar{A})$. We denote this as follows:

$(I ; M)-B E F O R E(\bar{A}) \Leftarrow \bar{M}(3,1)(T, T, T, \bar{T}, \bar{T}, \bar{A})$.

It is important to notice that $(I ; M)-B E F O R E$ applies to an I-wff and then yields an M-wff. The prefix $(I ; M)$ - is intended for this fact.

(b) $(I ; I)-B E F O R E(\bar{A}) \Leftarrow \bar{I}(4,1,2)(\bar{T}, \bar{T}, \bar{T}, \bar{T}, \bar{T}, \bar{A}, T, T, T, T)$.

(c) $(M ; I)-B E F O R E(A) \Leftarrow \bar{I}(3,1,2)(\bar{T}, \bar{T}, \bar{T}, T, T, A)$.

(d) $(M ; M)-B E F O R E(A) \Leftarrow \bar{M}(2,1)(T, A, \bar{T})$.

(2) Semantically,
(a) $\|(I ; M)-B E F O R E(\bar{A})\|_{t}=1$ iff $\left(\exists s_{1}\right)\left(\exists t_{1}\right)\left(t<s_{1}<t_{1} \wedge\|\bar{A}\|_{\left(s_{1}, t_{1}\right)}=1\right)$.
(b) $\|(I ; I)-B E F O R E(\bar{A})\|_{(s, t)}=1$ iff $\left(\exists s_{1}\right)\left(\exists t_{1}\right)\left(t<s_{1}<t_{1} \wedge\|\bar{A}\|_{\left(s_{1}, t_{1}\right)}=1\right)$.
(c) $\|(M ; I)-B E F O R E(A)\|_{(s, t)}=1$ iff $\left(\exists t_{1}\right)\left(t<t_{1} \wedge\|A\|_{t_{1}}=1\right)$.
(d) $\|(M ; M)-B E F O R E(A)\|_{t}=1$ iff $\left(\exists t_{1}\right)\left(t<t_{1} \wedge\|A\|_{t_{1}}=1\right)$.

Definition 4.1.2 (AFTER).

(1) (a) $(M ; I)-\operatorname{AFTER}(A) \Leftarrow \bar{I}(3,2,3)(\bar{T}, \bar{T}, \bar{T}, A, T, T)$.

(b) $(I ; I)-\operatorname{AFTER}(\bar{A}) \Leftarrow \bar{I}(4,3,4)(\bar{A}, \bar{T}, \bar{T}, \bar{T}, \bar{T}, \bar{T}, T, T, T, T)$.

(c) $(I ; M)-\operatorname{AFTER}(\bar{A}) \Leftarrow \bar{M}(3,3)(T, T, T, \bar{A}, \bar{T}, \bar{T})$.

(d) $(M ; M)-\operatorname{AFTER}(A) \Leftarrow \bar{M}(2,2)(A, T, \bar{T})$.

(2) Semantically,
(a) $\|(M ; I)-\operatorname{AFTER}(A)\|_{(s, t)}=1$ iff $\left(\exists t_{1}\right)\left(t_{1}<s \wedge\|A\|_{t_{1}}=1\right)$.
(b) $\|(\mathbb{I} ; I)-\operatorname{AFTER}(\bar{A})\|_{(s, t)}=1$ iff $\left(\exists s_{1}\right)\left(\exists t_{1}\right)\left(s_{1}<t_{1}<s \wedge\|\bar{A}\|_{\left(s_{1}, t_{1}\right)}=1\right)$.
(c) $\|(I ; M)-\operatorname{AFTER}(\bar{A})\|_{t}=1$ iff $\left(\exists s_{1}\right)\left(\exists t_{1}\right)\left(s_{1}<t_{1}<t \wedge\|\bar{A}\|_{\left(s_{1}, t_{1}\right)}=1\right)$.
(d) $\|(M ; M)-\operatorname{AFTER}(A)\|_{t}=1$ iff $\left(\exists t_{1}\right)\left(t_{1}<t \wedge\|A\|_{t_{1}}=1\right)$. 
Definition 4.1.3 (DURING).

(1) (a) $(I ; M)-D U R I N G_{1}(\bar{A}) \Leftarrow \bar{M}(2,2)(T, T, \bar{A})$.

(b) $(I ; M)-D U R I N G_{2}(\bar{A}) \Leftarrow \bar{M}(2,1)(T, T, \bar{A})$.

(c) $(I ; I)-D_{U R I N G}(\bar{A}) \Leftarrow \bar{I}(3,2,3)(\bar{T}, \bar{A}, \bar{T}, T, T, T)$.

(d) $(I ; I)-D U R I N G_{2}(\bar{A}) \Leftarrow \bar{I}(3,1,2)(\bar{T}, \bar{A}, \bar{T}, T, T, T)$.

(e) $(I ; M)-D U R I N G_{3}(\bar{A}) \leftarrow \bar{M}(3,2)(T, T, T, \bar{T}, \bar{A}, \bar{T})$.

(f) $(I ; I)-D U R I N G_{3}(\bar{A}) \Leftarrow \bar{I}(4,2,3)(\bar{T}, \bar{T}, \bar{A}, \bar{T}, \bar{T}, \bar{T}, T, T, T, T)$.

(2) Semantically,
(a) $\left\|(I ; M)-D U R I N G_{1}(\bar{A})\right\|_{t}=1$ iff $\left(\exists s_{1}\right)\left(s_{1}<t \wedge\|\bar{A}\|_{\left(s_{1}, t\right)}=1\right)$.
(b) $\left\|(I ; M)-D U R I N G_{2}(\bar{A})\right\|_{t}=1$ iff $\left(\exists t_{1}\right)\left(t<t_{1} \wedge\|\bar{A}\|_{\left(t, t_{1}\right)}=1\right)$.
(c) $\left\|(I ; I)-D U R I N G_{1}(\bar{A})\right\|_{(s, t)}=1$ iff $\left(\exists s_{1}\right)\left(s_{1}<s \wedge\|\bar{A}\|_{\left(s_{1}, t\right)}=1\right)$.
(d) $\left\|(I ; I)-D U R I N G_{2}(\bar{A})\right\|_{(s, t)}=1$ iff $\left(\exists t_{1}\right)\left(t<t_{1} \wedge\|\bar{A}\|_{\left(s, t_{1}\right)}=1\right)$.
(e) $\left\|(I ; M)-D U R I N G_{3}(\bar{A})\right\|_{t}=1$ iff $\left(\exists s_{1}\right)\left(\exists t_{1}\right)\left(s_{1}<t<t_{1} \wedge\|\bar{A}\|_{\left(s_{1}, t_{1}\right)}=1\right)$.
(f) $\left\|(I ; I)-D U R I N G_{3}(\bar{A})\right\|_{(s, t)}=1$ iff $\left(\exists s_{1}\right)\left(\exists t_{1}\right)\left(s_{1}<s \wedge t<t_{1} \wedge\|\bar{A}\|_{\left(s_{1}, t_{1}\right)}=1\right)$.

\section{Definition 4.1.4 (CONTAINS).}

(1) (a) (M;I)-CONTAINS $\operatorname{CO}_{1}(A) \Leftarrow \bar{I}(2,1,2)(\bar{T}, T, A)$.

(b) $(M ; I)-C O N T A I N S_{2}(A) \Leftarrow \bar{I}(2,1,2)(\bar{T}, A, T)$.

(c) $(I ; I)-C_{\text {CONTANS }}(\bar{A}) \Leftarrow \bar{I}(3,1,3)(\bar{T}, \bar{T}, \bar{A}, T, T, T)$.

(d) $(I ; I)-C O N T A I N S_{2}(\bar{A}) \Leftarrow \bar{I}(3,1,3)(\bar{A}, \bar{T}, \bar{T}, T, T, T)$.

(e) $(M ; I)-C O N \operatorname{TANS}_{3}(A) \Leftarrow \bar{I}(3,1,3)(\bar{T}, \bar{T}, \bar{T}, T, A, T)$.

(f) $(I ; I)-C O N T A I N S_{3}(\bar{A}) \Leftarrow \bar{I}(4,1,4)(\bar{T}, \bar{T}, \bar{T}, \bar{A}, \bar{T}, \bar{T}, T, T, T, T)$.

(2) Semantically,

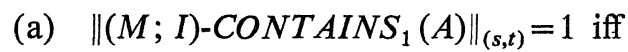
$\|A\|_{t}=1$.

(b) $\left\|(M ; I)-C O N T A I N S_{2}(A)\right\|_{(s, t)}=1$ iff $\|A\|_{s}=1$.

(c) $\left\|(I ; I)-C O N T A I N S_{1}(\bar{A})\right\|_{(s, t)}=1$ iff $\left(\exists s_{1}\right)\left(s<s_{1}<t \wedge\|\bar{A}\|_{\left(s_{1}, t\right)}=1\right)$. 
(d) $\left\|(I ; I)-C O N T A I N S_{2}(\bar{A})\right\|_{(s, t)}=1$ iff $\left(\exists t_{1}\right)\left(s<t_{1}<t \wedge\|\bar{A}\|_{\left(s, t_{1}\right)}=1\right)$.

(e) $\left\|(M ; I)-C O N T A I N S_{3}(A)\right\|_{(s, t)}=1$ iff $\left(\exists s_{1}\right)\left(s<s_{1}<t \wedge\|A\|_{s_{1}}=1\right)$.

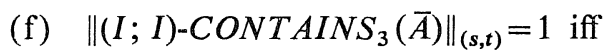
$\left(\exists s_{1}\right)\left(\exists t_{1}\right)\left(s<s_{1}<t_{1}<t \wedge\|\bar{A}\|_{\left(s_{1}, t_{1}\right)}=1\right)$.

We don't need any specific tense operators corresponding to Bruce's timesegment relation "same-time", but if the reader wants to, we can easily define them as follows:

Definition 4.1.5 (SAME-TIME).

(1) (a) $(I ; I)-S A M E-T I M E(\bar{A}) \Leftarrow \bar{I}(2,1,2)(\bar{A}, T, T)$.

(b) $(M ; M)-S A M E-T I M E(A) \Leftarrow \bar{M}(1,1)(A)$.

(2) Semantically,

(a) $\|(I ; I)-S A M E-T I M E(\bar{A})\|_{(s, t)}=1$ iff $\|\bar{A}\|_{(s, t)}=1$.

(b) $\|(M ; M)-S A M E-T I M E(A)\|_{t}=1$ iff $\|A\|_{t}=1$.

Definition 4.1.6 (OVERLAPS).

(1) (a) OVERLAPS $(\bar{A}) \leftarrow \bar{I}(4,1,3)(\bar{T}, \bar{T}, \bar{T}, \bar{T}, \bar{A}, \bar{T}, T, T, T, T)$.

(b) $\operatorname{OVERLAPS}_{2}(\bar{A}) \Leftarrow \bar{I}(3,1,2)(\bar{T}, \bar{T}, \bar{A}, T, T, T)$.

(2) Semantically,

(a) $\left\|O V E R L A P S_{1}(\bar{A})\right\|_{(s, t)}=1$ iff $\left(\exists s_{1}\right)\left(\exists t_{1}\right)\left(s<s_{1}<t<t_{1} \wedge\|\bar{A}\|_{\left(s_{1}, t_{1}\right)}=1\right)$.

(b) $\left\|O V E R L A P S_{2}(\bar{A})\right\|_{(s, t)}=1$ iff $\left(\exists t_{1}\right)\left(t<t_{1} \wedge\|\bar{A}\|_{\left(t, t_{1}\right)}=1\right)$.

Definition 4.1.7 (OVERLAPPED).

(1) (a) OVERLAPPED $D_{1}(\bar{A}) \Leftarrow \bar{I}(4,2,4)(\bar{T}, \bar{A}, \bar{T}, \bar{T}, \bar{T}, \bar{T}, T, T, T, T)$.

(b) OVERLAPPED $2(\bar{A}) \Leftarrow \bar{I}(3,2,3)(\bar{A}, \bar{T}, \bar{T}, T, T, T)$.

(2) Semantically,

(a) $\|$ OVERLAPPED $(\bar{A}) \|_{(s, t)}=1$ iff $\left(\exists s_{1}\right)\left(\exists t_{1}\right)\left(s_{1}<s<t_{1}<t \wedge\|\bar{A}\|_{\left(s_{1}, t_{1}\right)}=1\right)$.

(b) $\|$ OVERLAPPED $D_{2}(\bar{A}) \|_{(s, t)}=1$ iff $\left(\exists s_{1}\right)\left(s_{1}<s \wedge\|\bar{A}\|_{\left(s_{1}, s\right)}=1\right)$. 
It is easy to see that $(M ; M)-B E F O R E$ and $(M ; M)-A F T E R$ are no other than the Priorian tense operators $F$ and $P$.

Definition 4.1.8 $(F$ and $P$ ).

(1) (a) $F A \Leftarrow(M ; M)-B E F O R E(A)$.

(b) $P A \Leftarrow(M ; M)-\operatorname{AFTER}(A)$.

(2) Semantically,

(a) $\|F A\|_{t}=1$ iff $\left(\exists t_{1}\right)\left(t<t_{1} \wedge\|A\|_{t_{1}}=1\right)$.

(b) $\|P A\|_{t}=1$ iff $\left(\exists t_{1}\right)\left(t_{1}<t \wedge\|A\|_{t_{1}}=1\right)$.

The following tense operator is of some interest.

Definition 4.1.9 (CONTINUE).

(1) $\operatorname{CONTINUE}(A) \Leftarrow \neg(M ; I)-\operatorname{CONTAINS}_{3}(\neg A)$.

(2) Semantically, $\|\operatorname{CONTINUE}(A)\|_{(s, t)}=1$ iff $\left(\forall s_{1}\right)\left(s<s_{1}<t \rightarrow\|A\|_{s_{1}}=1\right)$.

By using the above operator CONTINUE together with $(I ; M)-D U R I N G_{3}$, we can define Scott's present progressive tense operator as follows:

Definition 4.1.10 (Scott's $O$ and the present progressive tense).

(1) (a) $O A \Leftarrow(I ; M)-D U R I N G_{3}(C O N T I N U E(A))$.

(b) $I N G(\bar{A}) \Leftarrow(I ; M)-D U R I N G_{3}(\bar{A})$.

(2) Semantically,

(a) $\|O A\|_{t}=1$ iff $\left(\exists s_{1}\right)\left(\exists t_{1}\right)\left(s_{1}<t<t_{1} \wedge\left(\forall s_{2}\right)\left(s_{1}<s_{2}<t_{1} \rightarrow\|A\|_{s_{2}}=1\right)\right)$.

(b) $\|I N G(\bar{A})\|_{t}=1$ iff $\left(\exists s_{1}\right)\left(\exists t_{1}\right)\left(s_{1}<t<t_{1} \wedge\|\bar{A}\|_{\left(s_{1}, t_{1}\right)}=1\right)$.

Now we deal with Kamp's since and until.

Definition 4.1.11 (Kamp's SINCE and UNTIL).

(1) (a) $K-\operatorname{SINCE}(A, B) \Leftarrow \bar{M}(2,2)(B, T, \operatorname{CONTINUE}(A))$.

(b) $K-U N T I L(A, B) \Leftarrow \bar{M}(2,1)(T, B, \operatorname{CONTINUE}(A))$.

(2) Semantically,

(a) $\|K-\operatorname{SINCE}(A, B)\|_{t}=1$ iff $(\exists s)\left(s<t \wedge\|B\|_{s}=1 \wedge\left(\forall s_{1}\right)\left(s<s_{1}<t \rightarrow\|A\|_{s_{1}}=1\right)\right)$.

(b) $\|K-U N T I L(A, B)\|_{t}=1$ iff $(\exists s)\left(t<s \wedge\|B\|_{s}=1\left(\forall s_{1}\right)\left(t<s_{1}<s \rightarrow\|A\|_{s_{1}}=1\right)\right)$. 
It seems to me that the second argument of since and until is usually an I-sentence. So the following definitions of since and until are no less plausible than the above.

Definition 4.1.12 (SINCE and UNTIL).

(1) (a) $\operatorname{SINCE}(A, \bar{B}) \Leftrightarrow \bar{M}(3,3)(T, T, T, \bar{B}, \bar{T}, \operatorname{CONTINUE}(A))$.

(b) $\operatorname{UNTIL}(A, \bar{B}) \Leftrightarrow \bar{M}(3,1)(T, T, T, \operatorname{CONTINUE}(A), \bar{T}, \bar{B})$.

(2) Semantically,

(a) $\|\operatorname{SINCE}(A, \bar{B})\|_{t}=1$ iff $\left(\exists s_{1}\right)\left(\exists s_{2}\right)\left(s_{1}<s_{2}<t \wedge\|\bar{B}\|_{\left(s_{1}, s_{2}\right)}=1 \wedge\left(\forall s_{3}\right)\left(s_{2}<s_{3}<t \rightarrow\|A\|_{s_{3}}=1\right)\right)$.

(b) $\|U N T I L(A, \bar{B})\|_{t}=1$ iff $\left(\exists s_{1}\right)\left(\exists s_{2}\right)\left(t<s_{1}<s_{2} \wedge\|\bar{B}\|_{\left(s_{1}, s_{2}\right)}=1 \wedge\left(\forall s_{3}\right)\left(t<s_{3}<s_{1} \rightarrow\|A\|_{s_{3}}=1\right)\right)$.

\section{§4.2. Tense and Aspect within IM}

The main purpose of this section is to give several paradigmatic analyses of tense and aspect within our formal framework IM. We give these analyses only to make the reader realize that such interval logics as given in this paper, if properly developed, will render a powerful tool to formal study of tense and aspect in English. Thus the reader should not take these analyses so seriously. They are incomplete and inadequate in several major respects. So they are to be regarded as a mere sketch or a mere illustration. The reader, who wants to develop interval logics for such a direction, is recommended to consult, say, Bennett and Partee (1978) and its bibliography, to which our basic idea owes much.

Let's begin with examples that can be treated well by traditional tense operators.

(1) Mary has been happy.

(2) Mary will be happy.

The analyses of these propositions go as follows:

(1a) $P p$, where $p=$ "Mary is happy".

(2a) $F p$, where $p=$ "Mary is happy".

But our analyses of the following propositions are radically different from the traditional ones.

(3) Tom has visited the museum. 
(4) Tom will buy a house.

(3a) $(I ; M)-\operatorname{AFTER}(\bar{p})$, where $\bar{p}=$ "Tom visits the museum".

(4a) $(I ; M)-\operatorname{AFTER}(\bar{p})$, where $\bar{p}=$ "Tom buys a house".

Now we deal with the progressive tenses.

(5) Susan is typing a letter.

(6) Mary has been building a house.

(7) Jane will be mastering English.

The following analyses of the above sentences are radically different from Scott's.

(5a) ING $(\bar{P})$, where $\bar{p}=$ "Susan types a letter".

(6a) $P(\operatorname{ING}(\bar{p}))$, where $\bar{p}=$ "Mary builds a house".

(7a) $F(I N G(\bar{p}))$, where $\bar{p}=$ "Jane masters English".

Now we consider several sentences with adverbian subordinate clauses headed by "while", "before", "after", etc.

(8) Tom read the newspaper before he took his family for a drive.

(8a) $\quad(I ; M)-\operatorname{AFTER}(\bar{p} \wedge(I ; I)-\operatorname{AFTER}(\bar{q}))$, where $\bar{p}=$ "Tom takes his family for a drive". $\bar{q}=$ "Tom reads the newspaper".

(9) Mary memorized the dialogue while Ruth went shopping.

(9a) $\quad(I ; M)-A F T E R\left(\bar{p} \wedge \operatorname{CONTAINS}_{3}(\bar{q})\right)$, where $\bar{p}=$ "Ruth goes shopping".

$\bar{q}=$ "Mary memorizes the dialogue".

(10) Jane took a walk while Susan was asleep.

(10a) $(I ; M)-A F T E R\left(C O N T I N E(p) \wedge C O N T A I N S_{3}(\bar{q})\right)$, where $p=$ "Susan is asleep". $\bar{q}=$ "Jane takes a walk".

(11) Carol had been happy until she got married with John.

(11a) $(I ; M)-\operatorname{AFTER}(\bar{p} \wedge \neg(M ; I)-\operatorname{AFTER}(\neg q))$, where $\bar{p}=$ "Carol gets married with John". $q=$ "Carol is happy".

(12) Carol has been unhappy since she met John.

(12a) $\operatorname{SINCE}(p, \bar{q})$, where $p=$ "Carol is unhappy". $\bar{q}=$ "Carol meets John". 
The adequate treatment of initiation, resumption, continuation, and cessation verb phrases is one of the most difficult problems in formal study of tense and aspect. Especially the adequate treatment of resumption verb phrases seems to require a broader notion of a sentence being true or false at a union of intervals of time, as Bennett and Partee has stressed. Therefore we should be satisfied here only with very crude analyses of the following sentences.

(13) Tom begins to hate Mary.

(14) Tom begins to run.

(15) Tom begins to master English.

(16) Tom continues to hate Mary.

(17) Tom continues to run.

(18) Tom continues to master English.

(19) Tom finishes hating Mary.

(20) Tom finishes running.

(21) Tom finishes mastering English.

First of all, we give the analyses in which the above sentences are regarded as I-sentences.

(13a) $(I ; I)-D U R I N G_{2}(C O N T I N U E(p)) \wedge$ OVERLAPPED $D_{2}(C O N T I N U E(\neg p))$, where $p=$ "Tom hates Mary".

(14a) $(I ; I)-D U R I N G_{2}(C O N T I N U E(I N G(\bar{p}))) \wedge$ OVERLAPPED $(C O N T I N U E(\neg I N G(\bar{p})))$, where $\bar{p}=$ "Tom runs".

(15a) $(I ; I)-D U R I N G_{2}(\bar{p})$, where $\bar{p}=$ "Tom masters English".

(16a) $(I ; I)-D U R I N G_{3}(C O N T I N U E(p))$, where $p=$ "Tom hates Mary".

(17a) $(I ; I)-D U R I N G_{3}(C O N T I N U E(I N G(\bar{p})))$, where $\bar{p}=$ "Tom runs".

(18a) $(I ; I)-D U R I N G_{3}(\bar{p})$, where $\bar{p}=$ "Tom masters English".

(19a) $(I ; I)-D U R I N G_{1}(C O N T I N U E(p)) \wedge$ OVERLAPS 2 (CONTINUE $(\neg p))$, where $p=$ "Tom hates Mary".

(20a) $(I ; I)-D U R I N G_{1}(C O N T I N U E(I N G(\bar{p}))) \wedge$ OVERLAPS ${ }_{2}(C O N T I N U E(\neg I N G(\bar{p})))$, where 
$\bar{p}=$ "Tom runs".

(21a) $(I ; I)-D U R I N G_{1}(\bar{p})$, where $\bar{p}=$ "Tom masters English".

Next we give the analyses of sentences (13)-(21) in which they are regarded as M-sentences.

(13b) $(I ; M)-D U R I N G_{2}(C O N T I N U E(p)) \wedge$

$(I ; M)-D U R I N G_{1}(C O N T I N U E(\neg p))$, where

$p=$ "Tom hates Mary".

(14b) $(I ; M)-D U R I N G_{2}(C O N T I N U E(I N G(\bar{p}))) \wedge$

$(I ; M)-D U R I N G_{1}(C O N T I N U E(\neg I N G(\bar{p})))$, where

$\bar{p}=$ "Tom runs".

(15b) $(I ; M)-D U R I N G_{2}(\bar{p})$, where

$\bar{p}=$ "Tom masters English".

(16b) $(I ; M)-D U R I N G_{3}(C O N T I N U E(p))$, where

$p=$ "Tom hates Mary".

(17b) $(I ; M)-D U R I N G_{3}(C O N T I N U E(I N G(\bar{p}))$, where $\bar{p}=$ "Tom runs".

(18b) $(I ; M)-D U R I N G_{3}(\bar{p})$, where $\bar{p}=$ "Tom masters English".

(19b) $(I ; M)-D U R I N G_{1}(C O N T I N U E(p)) \wedge$

$(I ; M)-D U R I N G_{2}(C O N T I N U E(\neg p))$, where

$p=$ "Tom hates Mary".

(20b) $(I ; M)-D U R I N G_{1}(C O N T I N U E(I N G(\bar{p}))) \wedge$

$(I ; M)-D U R I N G_{2}(C O N T I N U E(\neg I N G(\bar{p})))$, where

$\bar{p}=$ "Tom runs".

(21b) $(I ; M)-D U R I N G_{1}(\bar{p})$, where

$\bar{p}=$ "Tom masters English".

\section{Acknowledgement}

I wish to thank Professors Satoru Takasu, Masahiko Sato, and Takeshi Hayashi for their encouragements and discussions with me.

\section{References}

[1] Bennett, M. and Partee, B., Towards the logic of tense and aspect in English, 
unpublished draft (reproduced in [2] with postscript), 1972.

[2] - Towards the logic of tense and aspect in English, Indiana University Linguistics Club, 1978.

[3] Bruce, B. C., A model for temporal references and its applications in a question answering program, Artificial Intelligence, 3 (1972), 1-25.

[4] Cochiarella, N., Tense logic: a study of temporal reference, Ph. D. Thesis, 1966.

[ 5 ] Dowty, D. R., Towards a semantical analysis of verb aspect and the English 'imperfect' progressive, Linguistics and Philosophy, 1 (1977), 45-77.

[6] Gabbay, D. M., Model theory for tense logics, Annals of Math. Logic, 8 (1975), 185-236.

[7] - Investigations in modal and tense logics with applications to problems in philosophy and lignuistics, D. Reidel, 1976.

[ 8 ] Henkin, L. A., The completeness of the first-order functional calculus, J. Symbolic Logic, 14 (1949), 159-166.

[9] Kahn, K. and Gorry, G. A., Mechanizing temporal knowledge, Artificial Intelligence, 9 (1977), 87-108.

[10] Kamp, J. A. W., Tense logic and the theory of linear order, Ph. D. Thesis, 1968.

[11] Kim, J., Events as property exemplification, in Action theory (ed. by M. Brand and D. Walton), D. Reidel, 1976, 159-176.

[12] Lemmon, E. J. and Scott, D. S., Intensional logic, unpublished draft, 1966.

[13] Makinson, D., On some completeness theorems in modal logic, Zeitschrift für Mathematische Logik und Grundlagen der Mathematik, 12 (1966), 379-384.

[14] Monk, J. D., Mathematical logic, Springer-Verlag, 1976.

[15] Prior, A. N., Time and modality, Oxford, 1957.

[16] —- Past, present and future, Oxford, 1967.

[17] - Papers on time and tense, Oxford, 1968.

[18] Rescher, N. and Urquhart, A., Temporal logic, Springer-Verlag, 1971.

[19] Scott, D. S., Advice on modal logic, in Philosophical Problems in Logic (ed. by $\mathrm{K}$. Lambert), D. Reidel, 1970, 143-173.

[20] Shoenfield, J. A., Mathematical logic, Addison-Wesley, 1967.

[21] Sugihara, T., Temporal logic (in Japanese), Waseda University Press, 1974.

[22] Tanaka, K., The complete English grammar (in Japanese), Hakusuisha, 1953.

[23] von Wright, G. H., Norm and action, Routledge and Kegan Paul, 1963. 
